Discrete Comput Geom 28:49-73 (2002)

DOI: $10.1007 / \mathrm{s} 00454-001-0091-2$

\title{
Classification of Integral Expanding Matrices and Self-Affine Tiles*
}

\author{
Ibrahim Kirat ${ }^{1}$ and Ka-Sing Lau $^{2}$ \\ ${ }^{1}$ Department of Mathematics, Sakarya University, \\ Sakarya, Turkey \\ ibkst@yahoo.com \\ ${ }^{2}$ Department of Mathematics, The Chinese University of Hong Kong, \\ Shatin, New Territories, Hong Kong \\ kslau@math.cuhk.edu.hk
}

\begin{abstract}
Let $T$ be a self-affine tile that is generated by an expanding integral matrix $A$ and a digit set $D$. It is known that many properties of $T$ are invariant under the $\mathbb{Z}$ similarity of the matrix $A$. In [LW1] Lagarias and Wang showed that if $A$ is a $2 \times 2$ expanding matrix with $|\operatorname{det}(A)|=2$, then the $\mathbb{Z}$-similar class is uniquely determined by the characteristic polynomial of $A$. This is not true if $|\operatorname{det}(A)|>2$. In this paper we give complete classifications of the $\mathbb{Z}$-similar classes for the cases $|\operatorname{det}(A)|=3,4,5$. We then make use of the classification for $|\operatorname{det}(A)|=3$ to consider the digit set $D$ of the tile and show that $\mu(T)>0$ if and only if $\mathcal{D}$ is a standard digit set. This reinforces the conjecture in [LW3] on this.
\end{abstract}

\section{Introduction}

Let $A$ be an expanding integral matrix in $M_{n}(\mathbb{Z})$, i.e., all its eigenvalues $\lambda_{i}$ have modulus $>1$. Let $|\operatorname{det}(A)|=q$ and let $D=\left\{d_{1}, \ldots, d_{q}\right\} \subseteq \mathbb{Z}^{n}$ be a set of $q$ distinct vectors, called a $q$-digit set. The affine maps $w_{j}$ defined by

$$
w_{j}(x)=A^{-1}\left(x+d_{j}\right), \quad 1 \leq j \leq q,
$$

* I. Kirat was supported by a grant from the Sakarya University in Turkey. K.-S. Lau was supported by an RGC grant from Hong Kong and a direct grant from The Chinese University of Hong Kong. 
are all contractions under a suitable norm in $\mathbb{R}^{n}$ (see pp. 29-30 of [LW2]). The family $\left\{w_{j}\right\}_{j=1}^{q}$ is called an iterated function system (IFS) and there is a unique nonempty compact set satisfying $T=\bigcup_{j=1}^{q} w_{j}(T)[\mathrm{H}],[\mathrm{F}] . T$ is called the attractor of the system and is explicitly given by

$$
T:=T(A, D)=\left\{\sum_{i=1}^{\infty} A^{-i} d_{j_{i}}: d_{j_{i}} \in D\right\} .
$$

Let $\mu(T)$ denote the Lebesgue measure of $T$. We call $T$ an integral self-affine tile if $\mu(T)>0$. It is known that the measure $\mu(T)$ is an integer [LW3, Theorem 1.1], but in general it is difficult to determine if it is positive, in particular, if $\mu(T)=1$ [B], [GM], [LW1]-[LW5]. A natural approach for this is to consider the $\mathbb{Z}$-similarity of the matrices [LW4].

We say that $A, B \in M_{n}(\mathbb{Z})$ are $\mathbb{Z}$-similar, denoted by $A \sim B$, if there exists a unimodular matrix $P \in M_{n}(\mathbb{Z})$ (i.e., $P$ is invertible and $|\operatorname{det}(P)|=1$ ) such that $P A P^{-1}=$ $B . \mathbb{Z}$-similarity is an equivalence relationship and determines equivalence classes that are called $\mathbb{Z}$-similar classes. The measure problem $\mu(T)>0$ is invariant under $\mathbb{Z}$ similarity. Other properties of the tiles such as connectedness [BG], [GH], [HSV], [KL], the dimension of the boundary [KLSW], [SW], and the tiling problem [LW2] are all invariant under $\mathbb{Z}$-similarity.

The $\mathbb{Z}$-similarity classification of expanding integral matrices was first studied by Lagarias and Wang [LW1] using the characteristic polynomials (c.p.). They showed that each integral expanding polynomial (all roots have modulus > 1) $f(x)=x^{2}+a x+q$, $|q|=2$, corresponds to exactly one $\mathbb{Z}$-similar class of expanding matrices $A \in M_{2}(\mathbb{Z})$. Since there are six such polynomials, there are only six $\mathbb{Z}$-similar classes of $2 \times 2$ integral matrices with $|\operatorname{det}(A)|=2$.

In this paper we consider the classification problem of $A \in M_{2}(\mathbb{Z})$ with $|\operatorname{det}(A)|=$ $3,4,5$. The situation is considerably more complicated. For $|\operatorname{det}(A)|=3$, there are ten characteristic polynomials. We have

Theorem 1.1. Suppose $A \in M_{2}(\mathbb{Z})$ is expanding with $|\operatorname{det}(A)|=3$. Let $f(x)$ be the characteristic polynomial,

(i) if $f(x) \neq x^{2}+3$, then $A$ is $\mathbb{Z}$-similar to the companion matrix $C$ of $f(x)$,

(ii) if $f(x)=x^{2}+3$, then $A$ is $\mathbb{Z}$-similar to $\left[\begin{array}{rr}1 & 2 \\ -2 & -1\end{array}\right]$ or $C$.

For $|\operatorname{det}(A)|=4$ and 5, we summarize the results in the following two theorems. The details are in Theorems 4.1, 4.2 and 4.4 and Theorems 5.1 and 5.3.

Theorem 1.2. There are fourteen characteristic polynomials for the expanding matrices $A \in M_{2}(\mathbb{Z})$ with $|\operatorname{det}(A)|=4$. Four of them have unique $\mathbb{Z}$-similar classes; seven of them have two $\mathbb{Z}$-similar classes; for $f(x)=x^{2}-4$, there are three $\mathbb{Z}$-similar classes:

$$
\left[\begin{array}{cc}
2 & n \\
0 & -2
\end{array}\right] \quad \text { for } \quad n=0,1,2
$$


for $f(x)=(x \pm 2)^{2}$, there are infinitely many $\mathbb{Z}$-similar classes:

$$
\left[\begin{array}{cc}
\mp 2 & |n| \\
0 & \mp 2
\end{array}\right] \quad \text { for } \quad n \in \mathbb{Z}
$$

Theorem 1.3. There are eighteen characteristic polynomials for the expanding matrices $A \in M_{2}(\mathbb{Z})$ with $|\operatorname{det}(A)|=5$. Fourteen of them have unique $\mathbb{Z}$-similar classes. The other four are $x^{2} \pm 5$ and $x^{2} \pm 2 x+5$, each of them has two $\mathbb{Z}$-similar classes.

The proof of the theorems is inspired by [LW1] and [LW4]. First we observe from the theorem of Latimer and MacDuffee [N] that for a given irreducible integral monic polynomial $f(x)$ and a root $\theta$, there is a one-to-one correspondence between the ideal classes of the ring $\mathbb{Z}[\theta]$ and the $\mathbb{Z}$-similar classes of $A \in M_{n}(\mathbb{Z})$ that have $f(x)$ as c.p. For the classification of the ideals of $\mathbb{Z}[\theta]$, we make use of two approaches: one is to use the theorem of Latimer and MacDuffee and a table in [Mo] of the number of the ideal classes of $\mathbb{Z}[\theta]$ in the quadratic field $\mathbb{Q}[\theta]$; the other approach is to use a reduction scheme in [LW1] to reduce $A$ to $\mathbb{Z}$-similar matrices of a checkable form.

Let $A \in M_{n}(\mathbb{Z})$ be expanding with $|\operatorname{det}(A)|=q$ and let $D \subseteq \mathbb{Z}^{n}$ be a $q$-digit set. Let $L=Z[A, D]$ be the lattice generated by $D, A D, \ldots, A^{n-1} D . D$ is called a complete set of coset representatives of $L / A(L)$ if

$$
L=\bigcup_{i=1}^{q}\left(d_{i}+A(L)\right) \quad \text { and } \quad\left(d_{i}+A(L)\right) \cap\left(d_{j}+A(L)\right)=\emptyset \quad \text { for } \quad i \neq j .
$$

We say that $D$ is a standard digit set (with respect to $A$ ) if $D$ is a complete set of coset representatives of $L / A(L)$; otherwise, $D$ is called a nonstandard digit set. It is easy to show that if $q=2$, then $T$ is always a tile and the $D$ is standard. In [LW3] Lagarias and Wang proved that if $q$ is a prime and

$$
q \mathbb{Z}^{n} \nsubseteq A^{2}\left(\mathbb{Z}^{n}\right),
$$

then $\mu(T(A, D))>0$ if and only if $D$ is a standard digit set. They also conjecture that condition (1.1) is redundant [LW3]. We therefore consider the self-affine tiles for the case of $|\operatorname{det}(A)|=3$ that is not covered by (1.1):

$$
f(x)=x^{2} \pm 3 \quad \text { and } \quad x^{2} \pm 3 x+3 .
$$

Making use of Theorem 1.1 and a criterion of [LW3], we give an explicit expression of such digits and show that all the tiles are generated by standard digit sets. This reinforces the conjecture in [LW3].

We organize the paper as follows. In Section 2 we give the relevent results in number theory and some preliminary results concerning self-similar tiles. The classifications of Theorems 1.1, 1.2, and 1.3 are given in Sections 3, 4, and 5, respectively. In Section 6 we make use of the classification for $\operatorname{det}(A)=3$ to determine the digit sets $D$ such that the invariant set $T(A, D)$ is a tile. In Section 7 we make use of the classification to sort out those $A$ that are $\mathbb{Z}$-similar to the self-similar matrices, the most well-known class in the theory. The results of the paper are summarized as tables in the Appendix. 


\section{Preliminaries}

We first give two simple propositions that are used throughout the section.

Proposition 2.1. Let $A=\left[\begin{array}{ll}a & b \\ c & d\end{array}\right] \in M_{2}(\mathbb{Z})$. Then

(i) A is similar to

$$
\left[\begin{array}{cc}
a & -b \\
-c & d
\end{array}\right], \quad\left[\begin{array}{ll}
d & c \\
b & a
\end{array}\right], \quad\left[\begin{array}{cc}
d & -c \\
-b & a
\end{array}\right], \quad\left[\begin{array}{cc}
a+c & -a-c+b+d \\
c & d-c
\end{array}\right] .
$$

(ii) If $a, d \neq 0$ and $c= \pm 1$ or $b= \pm 1$, then $A \sim\left[\begin{array}{cc}0 & \times \\ \times & \times\end{array}\right]$.

Proof. (i) Take $P=\left[\begin{array}{rr}-1 & 0 \\ 0 & 1\end{array}\right],\left[\begin{array}{ll}0 & 1 \\ 1 & 0\end{array}\right],\left[\begin{array}{rr}0 & -1 \\ 1 & 0\end{array}\right],\left[\begin{array}{ll}1 & 1 \\ 0 & 1\end{array}\right]$, then $P A P^{-1}$ are the four matrices listed in the proposition.

(ii) For $c=1$, we take $P=\left[\begin{array}{ll}0 & 1 \\ 1 & d\end{array}\right]$; for $b=1$, we take $P=\left[\begin{array}{rr}-d & 1 \\ 1 & 0\end{array}\right]$. Then (i) concludes the proof.

Proposition 2.2. Let $A \in M_{2}(\mathbb{Z})$ with c.p. $f(x)=x^{2}-a x+q$, $q$ is a prime. Suppose $A \sim\left[\begin{array}{cc}0 & b_{12} \\ b_{21} & b_{22}\end{array}\right]$, then $A \sim C$, the companion matrix of $f(x)$.

Proof. By using $\mathbb{Z}$-similarity and comparing the c.p., it is easy to see that $b_{22}=$ $a, b_{12} b_{21}=-q$ so that $A \sim\left[\begin{array}{cc}0 & \pm 1 \\ \mp q & a\end{array}\right]$ and $\left[\begin{array}{cc}0 & \pm q \\ \mp 1 & a\end{array}\right]$. Also observe that $C=\left[\begin{array}{cc}0 & 1 \\ -q & a\end{array}\right]$ and $\sim\left[\begin{array}{rr}0 & q \\ -1 & a\end{array}\right]$ by taking $P=\left[\begin{array}{cc}-a & 1 \\ -1 & 0\end{array}\right]$. The lemma now follows from Proposition 2.1.

We will need some facts concerning the polynomials and the algebraic fields. The first one can be found in [KL].

Proposition 2.3. Let $f(x)=x^{m}+a_{m-1} x^{m-1}+\cdots \pm q$, where $a_{i} \in \mathbb{Z}$ and $q$ is a prime. Suppose all the roots of $f(x)$ have modulus $>1$, then $f(x)$ is irreducible in $\mathbb{Q}[x]$ and the roots are simple.

Let $R$ be a ring. We say that the two ideals $S$ and $T$ of $R$ are in the same class if there exist two nonzero elements $\alpha, \beta \in R$ such that $\alpha S=\beta T$. This relationship determines the ideal classes of $R$.

Theorem 2.4 (Latimer and MacDuffee $[\mathrm{N}]$ ). Let $f(x) \in \mathbb{Z}[x]$ be an irreducible monic polynomial of degree $n$ and let $\theta$ be a root of $f(x)$. Then there is a one-to-one correspondence between the ideal classes of the ring $\mathbb{Z}[\theta]$ and the $\mathbb{Z}$-similar classes of matrices $A \in M_{n}(\mathbb{Z})$ such that $f(A)=0$. 
Table 1. Class numbers.

\begin{tabular}{lc}
\hline \multicolumn{1}{c}{$m$} & $h_{m}$ \\
\hline $3,5,6,13,17,21,29,-1,-2,-3,-7,-11,-19$ & 1 \\
$-5,-15$ & 2 \\
\hline
\end{tabular}

Proposition 2.5 [Ma, p. 15]. Let $m$ be a square free integer and let $Q[\sqrt{m}]$ be the quadratic field (i.e., the rational field generated by $\sqrt{m}$ ). Then the set of algebraic integers $R$ in $Q[\sqrt{m}]$ is

$$
\begin{aligned}
& R=\{a+b \sqrt{m}: a, b \in \mathbb{Z}\} \quad \text { if } \quad m \equiv 2 \text { or } 3 \quad(\bmod 4) \\
& R=\left\{\frac{a+b \sqrt{m}}{2}: a, b \in \mathbb{Z}, a \equiv b \quad(\bmod 2)\right\} \quad \text { if } m \equiv 1 \quad(\bmod 4)
\end{aligned}
$$

The number of the ideal classes of the above rings $R \subseteq Q[\sqrt{m}]$ is tabled on pp. 313345 of [Mo]. In Table 1 we list those that we will need. Here $m$ is a square-free integer and $h_{m}$ is the class number.

Let $A \in M_{2}(\mathbb{Z})$ be an expanding matrix and let $f(x)$ be its c.p. Then the $\operatorname{root} \theta$ of $f(x)$ can be written as $\theta=\frac{1}{2}(u+v \sqrt{m}), u, v, m \in \mathbb{Z}$. In the next three sections we classify the $\mathbb{Z}$-similar classes of $A$ with $|\operatorname{det}(A)|=3,4,5$ using the following two methods:

Method I. If $f$ is irreducible and $\mathbb{Z}[\theta]=R$ as in (2.1) or (2.2), then we can determine the number of $\mathbb{Z}$-similar classes of $f$ by applying the theorem of Latimer and MacDuffee and Table 1.

Method II. If the above conditions on $f$ are not satisfied, then we use the following scheme from [LW1]. Let $A=\left[\begin{array}{ll}a_{11} & a_{12} \\ a_{21} & a_{22}\end{array}\right]$ and let $p(A):=-a_{11} a_{22}$.

(a) If $p(A)>0$, we consider the unimodular matrices $P=\left[\begin{array}{cc}1 & -\varepsilon \\ 0 & 1\end{array}\right]$ and $P^{\prime}=$ $\left[\begin{array}{cc}1 & 0 \\ -\varepsilon^{\prime} & 1\end{array}\right]$ where $\varepsilon:=\operatorname{sign}\left(a_{11} a_{21}\right), \varepsilon^{\prime}:=\operatorname{sign}\left(a_{22} a_{12}\right)$. Let $A_{1}=P A P^{-1}$ or $P^{\prime} A\left(P^{\prime}\right)^{-1}$, then

$$
p\left(A_{1}\right)=p(A)+a_{21}^{2}+\varepsilon a_{21}\left(a_{22}-a_{11}\right)
$$

or, respectively

$$
p\left(A_{1}\right)=p(A)+a_{12}^{2}+\varepsilon^{\prime} a_{12}\left(a_{11}-a_{22}\right) .
$$

We aim to have $p\left(A_{1}\right)<p(A)$, so as to reduce $A$ to a $\mathbb{Z}$-similar matrix with smaller $p(\cdot)$. We can repeat this $k$ times to obtain a $\mathbb{Z}$-similar matrix $A_{k}$ with $p\left(A_{k}\right)$ equal to a few specific cases.

(b) For all these specific cases, we determine their $\mathbb{Z}$-similar classes individually. 
The following proposition enables us to list all the expanding characteristic polynomials of degree 2 [BG].

Proposition 2.6. Suppose $A \in M_{2}(\mathbb{Z})$ has c.p. $f(x)=x^{2}+$ ax+q with a, $q \in \mathbb{Z}, q \neq$ 0 . Then $A$ is expanding if and only if $|a| \leq q$ for $q>0$ and $|a| \leq|q|-2$ for $q<0$.

The following lemma is easy to prove [KL].

\section{Lemma 2.7 .}

(a) Let $p(x)$ and $\tilde{p}(x)$ denote the characteristic polynomials of $A,-A \in M_{n}(\mathbb{Z})$, respectively. Then $\tilde{p}(x)=(-1)^{n} p(-x)$.

(b) Let $A \in M_{n}(\mathbb{Z})$ be an expanding matrix with $|\operatorname{det}(A)|=q$ and let $D=$ $\left\{d_{1}, \ldots, d_{q}\right\} \subseteq \mathbb{R}^{n}$. Suppose there exists a vector $v \in \mathbb{R}^{n}$ such that $D=v-D$. Then $T(-A, D)=T(A, D)-\left(A-A^{-1}\right)^{-1} v$.

Remark. By the lemma we only need to consider one of the expanding polynomials $f(x)=x^{2} \pm a x+q$ in the $\mathbb{Z}$-classification.

\section{Expanding Matrices with Determinant \pm 3}

For an expanding matrix $A \in M_{2}(\mathbb{Z})$ with $|\operatorname{det}(A)|=3$, it is easy to obtain from Proposition 2.6 that the c.p. $f(x)$ is one of the following:

(I) $x^{2}-3, \quad x^{2} \pm 2 x+3$,

(II) $x^{2} \pm x-3, \quad x^{2} \pm x+3, \quad x^{2} \pm 3 x+3$,

(III) $x^{2}+3$.

Theorem 3.1. Suppose an expanding matrix $A \in M_{2}(\mathbb{Z})$ has c.p. $f(x)$ in (I) or (II). Then $A$ is $\mathbb{Z}$-similar to the companion matrix $C$ of $f(x)$.

Proof. The polynomial $f(x)=x^{2}-3$ has a root $\theta=\sqrt{3}$ and $\mathbb{Z}[\theta]$ is of the form (2.1). Proposition 2.5 asserts that $\mathbb{Z}[\theta]$ is the set of algebraic integers in the quadratic field $Q[\sqrt{3}]$, and it has only one ideal class (see Table 1). Therefore, there is only one similarity class for $f$ by Theorem 2.4 , and it is represented by $C$.

For $f(x)=x^{2} \pm 2 x+3$ in (I), we can consider the roots $\theta= \pm 1+\sqrt{-2}$ and the same argument applies.

For the polynomials in (II), we consider the respective roots $\theta=\frac{1}{2}( \pm 1+\sqrt{13}), \frac{1}{2}( \pm 1$ $+\sqrt{-11}), \frac{1}{2}( \pm 3+\sqrt{-3})$. In each of the cases, $R=Z[\theta]$ is of the form (2.2). By Proposition 2.5, they are the sets of algebraic integers in the quadratic fields $Q[\sqrt{-3}]$, $Q[\sqrt{-11}], Q[\sqrt{13}]$, respectively. Again there is only one ideal class of $\mathbb{Z}[\theta]$ from Table 1 and Theorem 2.4 applies.

We remark that for $x^{2}+3, \theta=\sqrt{-3}$ is a root, and $\mathbb{Z}[\theta]=\{a+b \sqrt{-3}: a, b \in \mathbb{Z}\}$ is not of the form $R$ in (2.1) and (2.2). Hence the table given for such $R$ does not 
necessarily give the number of ideal classes of $\mathbb{Z}[\theta]$. To determine the $\mathbb{Z}$-similar classes, we use Method II as outlined in the last section.

Lemma 3.2. Let $A=\left[a_{i j}\right] \in M_{2}(\mathbb{Z})$ be expanding and have c.p. $f(x)=x^{2}+3$. Then $a_{11}=-a_{22}$ and $p(A)=a_{11}^{2} \geq 0$. In the case $p(A)>0$, we have $0<\left|a_{21}\right| \leq\left|a_{11}\right|+1$ or $0<\left|a_{12}\right| \leq\left|a_{11}\right|$.

Proof. Since $f(x)=x^{2}+3=x^{2}-\left(a_{11}+a_{22}\right) x+\operatorname{det}(A)$, it follows that $a_{11}+a_{22}=0$ so that $p(A)=a_{11}^{2} \geq 0$. To prove the second part, we first observe that $a_{12}, a_{21} \neq 0$. Otherwise, we will have $3=\operatorname{det}(A)=a_{11} a_{22}=-a_{11}^{2}$ which is impossible. For the remaining inequalities, we assume the contrary holds, i.e., $\left|a_{21}\right|>\left|a_{11}\right|+1,\left|a_{12}\right|>\left|a_{11}\right|$, then

$$
\left|a_{12} a_{21}\right| \geq\left(\left|a_{11}\right|+2\right)\left(\left|a_{11}\right|+1\right)=\left|a_{11}\right|^{2}+3\left|a_{11}\right|+2 .
$$

It follows that $3=|\operatorname{det} A| \geq\left|a_{12} a_{21}\right|-\left|a_{11}\right|^{2} \geq 5$ and is impossible.

Theorem 3.3. Suppose $A=\left[a_{i j}\right] \in M_{n}(\mathbb{Z})$ and has c.p. $f(x)=x^{2}+3$, then $A$ is either $\mathbb{Z}$-similar to the companion matrix $C$ or $\left[\begin{array}{rr}1 & 2 \\ -2 & -1\end{array}\right]$.

Proof. It is clear that the two matrices above have c.p. $f(x)=x^{2}+3$. To show that they are not $\mathbb{Z}$-similar, we let $P=\left[\begin{array}{ll}a & b \\ c & d\end{array}\right]$ with $\operatorname{det}(P)=a d-b c=1$ (same argument for -1$)$. Then $P^{-1}=\left[\begin{array}{rr}d & -b \\ -c & a\end{array}\right]$. A direct computation shows that $P\left[\begin{array}{rr}1 & 2 \\ -2 & -1\end{array}\right] P^{-1}=$ $\left[\begin{array}{cc}d(a-2 b)-c(2 a-b) & \times \\ \times & \times\end{array}\right]$. Note that $d(a-2 b)-c(2 a-b)=1+2(b c-d b-a c) \neq 0$. It follows that $\left[\begin{array}{rr}1 & 2 \\ -2 & -1\end{array}\right]$ and $C=\left[\begin{array}{rr}0 & 1 \\ -3 & 0\end{array}\right]$ are not $\mathbb{Z}$-similar.

Now to show that there are only two similarity classes, we adopt Method II of the previous section. Lemma 3.2 says that $p(A) \geq 0$. For $p(A)=0$, we have $A \sim C$ by Proposition 2.2. For $p(A)>0$, we divide our consideration into two cases:

(i) If $\left|a_{11}\right|=1, A$ is $\mathbb{Z}$-similar to $\left[\begin{array}{rr}1 & 2 \\ -2 & -1\end{array}\right]$ or $\left[\begin{array}{rr}1 & 4 \\ -1 & -1\end{array}\right]$ (and their $\mathbb{Z}$-similar variations as in Proposition 2.1). The second matrix is $\mathbb{Z}$-similar to $\left[\begin{array}{ll}0 & x \\ x & \times\end{array}\right]$ by Proposition 2.1, and hence to $C$ by Proposition 2.2.

(ii) If $\left|a_{11}\right|>1$, we consider the two cases in Lemma 3.2. For $0<\left|a_{21}\right| \leq\left|a_{11}\right|+1$, by observing that $a_{11}=-a_{22}$, we can rewrite (2.3) as

$$
p\left(A_{1}\right)=p(A)+a_{21}^{2}+\varepsilon a_{21}\left(a_{22}-a_{11}\right)=p(A)+a_{21}^{2}-2\left|a_{21} \| a_{11}\right| .
$$

It is elementary to show that $p\left(A_{1}\right)<p(A)$. For the other case, $0<\left|a_{12}\right| \leq\left|a_{11}\right|$, we can draw the same conclusion by using the alternative form $(2.3)^{\prime}: p\left(A_{1}\right)=p(A)+$ $a_{12}^{2}+\varepsilon^{\prime} a_{12}\left(a_{11}-a_{22}\right)$.

We continue this process to construct $\mathbb{Z}$-similar matrices $A_{k}$ with decreasing $p\left(A_{k}\right)$ until $p\left(A_{k}\right)=0$ or $p\left(A_{k}\right)=1$ as in case (i), then we conclude that $f(x)$ only have the two $\mathbb{Z}$-similar classes as listed. 


\section{Expanding Matrices with Determinant \pm 4}

By Proposition 2.6, there are 14 possible c.p.'s for the expanding matrices $A \in M_{2}(\mathbb{Z})$ with $|\operatorname{det}(A)|=4$. They are

(I) $x^{2} \pm 3 x+4, \quad x^{2} \pm x-4$,

(II) $x^{2}+4, \quad x^{2} \pm x+4, \quad x^{2} \pm 2 x+4, \quad x^{2} \pm 2 x-4$,

(III) $x^{2}-4, \quad x^{2} \pm 4 x+4$.

Theorem 4.1. Each A with c.p. in (I) is $\mathbb{Z}$-similar to its companion matrix $C$.

Proof. For the two set of polynomials in (I), we consider the respective roots $\theta=$ $\frac{1}{2}( \pm 3+\sqrt{-7}), \frac{1}{2}( \pm 1+\sqrt{17})$. Then $\mathbb{Z}[\theta]=R$ are of the form (2.2) and hence are the set of algebraic integers in the quadratic fields $Q[\sqrt{-7}], Q[\sqrt{17}]$, respectively, by Proposition 2.5. From Table 1, there is only one ideal class of $R=Z[\theta]$. The conclusion follows by Theorem 2.4 .

Theorem 4.2. Each c.p. in (II) has two $\mathbb{Z}$-similarity classes of expanding matrices. The representatives of these classes, besides the companion matrix $C$, are listed according to the ordering in (II) as $\left[\begin{array}{rr}0 & 2 \\ -2 & 0\end{array}\right],\left[\begin{array}{rr} \pm 1 & 2 \\ -3 & \mp 2\end{array}\right],\left[\begin{array}{rr}0 & 2 \\ -2 & \mp 2\end{array}\right],\left[\begin{array}{rr}0 & 2 \\ 2 & \mp 2\end{array}\right]$.

Proof. It is direct to check that the list of matrices has the corresponding c.p.'s in (II). To check that they are not $\mathbb{Z}$-similar to the companion matrices, we only try the case $x^{2}-x+4$ where $A \sim\left[\begin{array}{ll}-1 & 2 \\ -3 & 2\end{array}\right]$ and $C=\left[\begin{array}{rr}0 & 1 \\ -4 & 1\end{array}\right]$. The others will follow from the same pattern of proof. We let $P=\left[\begin{array}{ll}a & b \\ c & d\end{array}\right]$ with $\operatorname{det}(P)=a d-b c= \pm 1$. If $C P=P A$, then we have $\left[\begin{array}{ll}c & d \\ \times & \times\end{array}\right]=\left[\begin{array}{rr}-a-3 b & 2(a+b) \\ \times & \times\end{array}\right]$. By comparing the two columns and plugging in $a d-b c=$ \pm 1 , we have $a=\frac{1}{4}\left(-3 b \pm \sqrt{-15 b^{2} \pm 8}\right) \notin \mathbb{Z}$. This is a contradiction, and $A \ngtr C$.

Now we show that the c.p. has exactly two $\mathbb{Z}$-similarity classes. We first consider $x^{2} \pm x+4$. The respective roots are $\theta=\frac{1}{2}(\mp 1+\sqrt{-15})$ and $\mathbb{Z}[\theta]=R$ as in (2.2). Hence $\mathbb{Z}[\theta]$ is the set of algebraic integers in the quadratic field $Q[\sqrt{-15}]$, by Proposition 2.5. By Table 1, there are two ideal classes of $\mathbb{Z}[\theta]$.

For the rest of the cases, Method I does not apply and we use the lengthier Method II in each case:

(A) $x^{2}+4$. The proof is identical to the case $x^{2}+3$ in Theorem 3.3. We only need to provide a check for the case $\left|a_{11}\right|=0,1,2$ as in the proof of case (i) in Theorem 3.3: If $\left|a_{11}\right|=0$, it can be shown that there are two $\mathbb{Z}$-similar classes: $C,\left[\begin{array}{rr}0 & 2 \\ -2 & 0\end{array}\right]$. Now suppose that $\left|a_{11}\right|=1$. It is easy to derive that the only case is $A \sim\left[\begin{array}{cr} \pm 1 & 5 \\ -1 & \mp 1\end{array}\right]$ (and its $\mathbb{Z}$-similar variations as in Proposition 2.1). By Proposition 2.1, $A \sim B$ with $b_{11}=0$. In the case $\left|a_{11}\right|=2$, we have the possibilities $A=\left[\begin{array}{cc}2 & \pm 8 \\ \mp 1 & -2\end{array}\right],\left[\begin{array}{cc}2 & \pm 4 \\ \mp 2 & -2\end{array}\right]$ (and their $\mathbb{Z}$-similar variations as in Proposition 2.1). In all cases, by Proposition 2.1, $A \sim B$ with $b_{11}=0$, which is the first case. 
(B) $x^{2} \pm 2 x+4, x^{2} \pm 2 x-4$. We only consider the case $x^{2}-2 x+4$. Firstly, we make a modification of Proposition 2.2: Suppose $A \sim B$ with $b_{11}=0$, then $b_{22}=2$, $\left(b_{12}, b_{21}\right)=( \pm 1, \mp 4),( \pm 4, \mp 1),( \pm 2, \mp 2)$. It is easy to check by Proposition 2.1 that there are two $\mathbb{Z}$-similar classes: $C=\left[\begin{array}{rr}0 & 1 \\ -4 & 2\end{array}\right]$ and $\left[\begin{array}{rr}0 & 2 \\ -2 & 2\end{array}\right]$.

We now try to make use of $p(A)=-a_{11} a_{22}$ to reduce $A$ to a $\mathbb{Z}$-similar matrix $B$ with $b_{11}=0$. Since the middle term of the c.p. implies that $a_{11}+a_{22}=2$, we have $4=a_{11}^{2}+2 a_{11} a_{22}+a_{22}^{2} \geq 4 a_{11} a_{22}$ and hence $p(A)=-a_{11} a_{22} \geq-1$. We check on each case:

(i) If $p(A)=-1$, then $a_{11} a_{22}=1$ and $a_{12} a_{21}=-3$. It follows that $a_{11}=a_{22}=1$ and one of the $a_{12}$ or $a_{21}$ equals \pm 1 . We can use Proposition 2.1 to transform $A$ into a $\mathbb{Z}$-similar $B$ with $b_{11}=0$.

(ii) If $p(A)=0$, then $A$ is already in the form of $B$.

(iii) If $p(A)>0$ and $\left|a_{11}\right|=1$, then $a_{11} a_{22}=-3, a_{12} a_{21}=-7$. The same argument as in (i) applies.

(iv) If $p(A)>0$ and $\left|a_{11}\right|=2$, we claim that $a_{11} \neq 2$. Otherwise, by comparing the middle term of the c.p., we have $a_{22}=0$ so that $p(A)=0$. Hence we only have $a_{11}=-2$ and $a_{22}=4$, and $a_{12} a_{21}=-p(A)-\operatorname{det}(A)=-12$. We have the following possibilities for $A:\left[\begin{array}{rr}-2 & \pm 12 \\ \mp 1 & 4\end{array}\right],\left[\begin{array}{rr}-2 & \pm 6 \\ \mp 2 & 4\end{array}\right],\left[\begin{array}{rr}-2 & \pm 4 \\ \mp 3 & 4\end{array}\right]$, and their transposes. By Proposition 2.1, they can all be reduced to the form of $B$ with $b_{11}=0$.

(v) If $p(A)>0$ and $\left|a_{11}\right|=3$, we have $\left(a_{11}, a_{22}\right)=(3,-1),(-3,5)$. Then $a_{12} a_{21}=-p(A)-\operatorname{det}(A)=-7,-19$. We have the following possibilities for $A$ : $\left[\begin{array}{rr}3 & \pm 7 \\ \mp 1 & -1\end{array}\right],\left[\begin{array}{rr}-3 & \pm 19 \\ \mp 1 & 5\end{array}\right]$, and their transposes. Again they can be transformed into the required $B$ by Proposition 2.1 .

(vi) If $p(A)>0$ and $\left|a_{11}\right|=4$, we have $\left(a_{11}, a_{22}\right)=(4,-2),(-4,6)$. Then $a_{12} a_{21}=-p(A)-\operatorname{det}(A)=-12,-28$. We have the following possibilities for $A:\left[\begin{array}{rr}4 & \pm 12 \\ \mp 1 & -2\end{array}\right],\left[\begin{array}{rr}4 & \pm 6 \\ \mp 2 & -2\end{array}\right],\left[\begin{array}{rr}4 & \pm 4 \\ \mp 3 & -2\end{array}\right],\left[\begin{array}{rr}-4 & \pm 28 \\ \mp 1 & 6\end{array}\right],\left[\begin{array}{rr}-4 & \pm 14 \\ \mp 2 & 6\end{array}\right],\left[\begin{array}{rr}-4 & \pm 7 \\ \mp 4 & 6\end{array}\right]$, and their transposes. They can be transformed into the required $B$ by Proposition 2.1. To see $\left[\begin{array}{rr}-4 & \pm 14 \\ \mp 2 & 6\end{array}\right] \sim$ $\left[\begin{array}{rr}0 & -2 \\ 2 & 2\end{array}\right]$, one needs to apply the last similarity in Proposition 2.1(i) (twice).

(vii) If $p(A)>0$ and $\left|a_{11}\right| \geq 5$, we can use the same argument as in Lemma 3.2 to show that either $0<\left|a_{12}\right| \leq\left|a_{22}\right|$ or $0<\left|a_{21}\right| \leq\left|a_{11}\right|+1$. For $0<\left|a_{21}\right| \leq\left|a_{11}\right|+1$, we let $\left|a_{11}\right|=1+l, l \geq 4$. Then

$$
\begin{aligned}
p\left(A_{1}\right) & =p(A)+a_{21}^{2}+\varepsilon a_{21}\left(2-2 a_{11}\right) \\
& =p(A)+\left(a_{21}^{2}-\left|a_{21} \| a_{11}\right|-\left|a_{21}\right|\right)+\left(2 \varepsilon a_{21}-l\left|a_{21}\right|\right) \\
& <p(A) .
\end{aligned}
$$

For $0<\left|a_{12}\right| \leq\left|a_{22}\right|$, we let $\left|a_{22}\right|=2+k, k \geq 1$ (since $\left|a_{11}\right| \geq 5$ ), then using the alternate expression (2.3) of $p(A)$, we have

$$
\begin{aligned}
p\left(A_{1}\right) & =p(A)+a_{12}^{2}+\varepsilon^{\prime} a_{12}\left(2-2 a_{22}\right) \\
& =p(A)+\left(a_{12}^{2}-\left|a_{12} \| a_{22}\right|\right)+\left(2 \varepsilon^{\prime} a_{12}-(2+k)\left|a_{12}\right|\right) \\
& <p(A) .
\end{aligned}
$$


In either case we can reduce $A$ to a $\mathbb{Z}$-similar matrix $A_{k}$ in the form of (i)-(vi) and the theorem follows.

It remains to consider the c.p. in group (III).

\section{Lemma 4.3.}

(i) $\left[\begin{array}{rr}2 & m \\ 0 & -2\end{array}\right] \sim\left[\begin{array}{rr}2 & n \\ 0 & -2\end{array}\right]$ if and only if $\left.n \equiv \pm m \bmod 4\right)$,

(ii) $\left[\begin{array}{rr}2 & m \\ 0 & 2\end{array}\right] \sim\left[\begin{array}{ll}2 & n \\ 0 & 2\end{array}\right]$ if and only if $|m|=|n|$.

Proof. (i) To prove the first assertion, we let $P=\left[\begin{array}{ll}a & b \\ c & d\end{array}\right]$ with $\operatorname{det}(P)=a d-b c= \pm 1$. If they were $\mathbb{Z}$-similar, then $P\left[\begin{array}{cc}2 & n \\ 0 & -2\end{array}\right]=\left[\begin{array}{cc}2 & m \\ 0 & -2\end{array}\right] P$. This reduces to $\left[\begin{array}{cc}2 a & n a-2 b \\ 2 c & n c-2 d\end{array}\right]=$ $\left[\begin{array}{cc}2 a+m c & 2 b+m d \\ -2 c & -2 d\end{array}\right]$. It follows that $c=0, a d= \pm 1$, and $n a=4 b+m d$. Hence, we must have $n \equiv \pm m \bmod 4)$. Conversely, for $n \equiv \pm m \bmod 4)$, we can take $P=\left[\begin{array}{cc}1 & \frac{1}{4}(n \mp m) \\ 0 & \pm 1\end{array}\right]$ So that $\left[\begin{array}{cc}2 & n \\ 0 & -2\end{array}\right] \sim\left[\begin{array}{cc}2 & m \\ 0 & -2\end{array}\right]$.

(ii) Let $P=\left[\begin{array}{ll}a & b \\ c & d\end{array}\right]$ with $\operatorname{det}(P)=a d-b c= \pm 1$. Then $\left[\begin{array}{ll}2 & n \\ 0 & 2\end{array}\right]=P\left[\begin{array}{cc}2 & m \\ 0 & 2\end{array}\right] P^{-1}$ $=\left[\begin{array}{cc}\mp a c m+2 & \pm a^{2} m \\ \mp c^{2} m & \pm a c m+2\end{array}\right]$ implies that $\pm a^{2} m=n$ and $\mp c^{2} m=0$. Thus if $m=0$, then $n=0$ and (ii) follows. If $m \neq 0$, then $c=0$ so that $\pm 1=a d-b c=a d$. Therefore, $a= \pm 1$ implies $n= \pm a^{2} m= \pm m$. The sufficiency follows from Proposition 2.1.

Theorem 4.4. For $x^{2}-4$, the $\mathbb{Z}$-similar classes are $\left[\begin{array}{cc}2 & n \\ 0 & -2\end{array}\right]$ for $n=0,1,2$; and for $x^{2} \pm 4 x+4$, they are $\left[\begin{array}{rr}\mp 2 & |n| \\ 0 & \mp 2\end{array}\right]$ for all $n \in \mathbb{Z}$.

Proof. For $A$ with characteristic polynomial $p(x)=x^{2}-4$ or $(x-2)^{2}$, let $v=\left[\begin{array}{l}x \\ y\end{array}\right]$ be an eigenvector of 2 , then $v$ can be chosen as a vector with integral entries $x, y$ such that $\operatorname{gcd}(x, y)=1$. Hence, there exist $m, m^{\prime} \in \mathbb{Z}$ such that $x m-y m^{\prime}=1$. Let $P=\left[\begin{array}{cc}x & m^{\prime} \\ y & m\end{array}\right]$. Then a direct calculation shows that $P^{-1} A P=\left[\begin{array}{cc}m & -m^{\prime} \\ -y & x\end{array}\right] A\left[\begin{array}{cc}x & m^{\prime} \\ y & m\end{array}\right]=\left[\begin{array}{cc}2 & n \\ 0 & \pm 2\end{array}\right]$ for some $n \in \mathbb{Z}$. We note that, by Proposition 2.1, $\left[\begin{array}{cc}2 & n \\ 0 & \pm 2\end{array}\right] \sim\left[\begin{array}{cc}2 & -n \\ 0 & \pm 2\end{array}\right]$. Also, using $P=\left[\begin{array}{rr}1 & 1 \\ 0 & -1\end{array}\right]$, we get $\left[\begin{array}{rr}2 & 1 \\ 0 & -2\end{array}\right] \sim\left[\begin{array}{rr}2 & 3 \\ 0 & -2\end{array}\right]$. Then Lemma 4.3 completes the first part of the proof.

For $A$ with characteristic polynomial $p(x)=(x+2)^{2}$, we proceed as above with $v$ being an eigenvector of -2 so that $A \sim\left[\begin{array}{rr}-2 & n \\ 0 & -2\end{array}\right]$ for some $n \in \mathbb{Z}$. We again use Lemma 4.3 to conclude the proof.

Remark. The argument in the above proof works for any quadratic characteristic polynomial with an integer eigenvalue $\lambda$ of multiplicity 2 . 


\section{Expanding Matrices with Determinant \pm 5}

By Proposition 2.6, we can write down the 18 possible c.p.'s $f(x)$ for the expanding matrices $A \in M_{2}(\mathbb{Z})$ with $|\operatorname{det} A|=5$. They are

(I) $x^{2} \pm 3 x-5, \quad x^{2} \pm 2 x-5, \quad x^{2} \pm x-5, \quad x^{2} \pm x+5, \quad x^{2} \pm 3 x+5$, $x^{2} \pm 4 x+5, \quad x^{2} \pm 5 x+5$

(II) $x^{2} \pm 5, \quad x^{2} \pm 2 x+5$.

Theorem 5.1. Suppose an expanding matrix $A \in M_{2}(\mathbb{Z})$ has c.p. $f(x)$ in (I). Then $A$ is $\mathbb{Z}$-similar to the companion matrix of $f(x)$.

We omit the proof because it is similar to that of Theorem 3.1. Our main proof is for the polynomials in group (II).

Lemma 5.2. Let $A \in M_{2}(\mathbb{Z})$ be expanding with c.p. $f(x)=x^{2}+a x \pm 5$. If $p(A)>0$, then either $0<\left|a_{12}\right| \leq\left|a_{22}\right|$ or $0<\left|a_{21}\right| \leq\left|a_{11}\right|+2$.

Proof. Note that $a_{12}, a_{21} \neq 0$. Otherwise, $a_{12} a_{21}=0$ implies that $\pm 5=\operatorname{det} A=$ $a_{11} a_{22}$, and $a_{11}$ and $a_{22}$ are eigenvalues of $A$. It follows that one of the eigenvalues has modulus 1 , which contradicts that $A$ is expanding.

The inequality can be proved as in the proof of Lemma 3.2.

Theorem 5.3. $\quad$ Each c.p. $f(x)$ in (II) corresponds to two $\mathbb{Z}$-similar classes. Besides the companion matrix, the representing matrices of the four polynomials are listed as follows according to the order in (II):

$$
\left[\begin{array}{rr}
2 & 3 \\
-3 & -2
\end{array}\right],\left[\begin{array}{rr}
1 & 2 \\
2 & -1
\end{array}\right],\left[\begin{array}{rr}
-1 & 2 \\
-2 & -1
\end{array}\right],\left[\begin{array}{rr}
1 & 2 \\
-2 & 1
\end{array}\right]
$$

Proof. We claim that the matrices listed are not $\mathbb{Z}$-similar to the corresponding companion matrices. To check the first one we let $P=\left[\begin{array}{ll}a & b \\ c & d\end{array}\right]$ with $\operatorname{det}(P)=1$ (or -1). Then for $A=\left[\begin{array}{rr}2 & 3 \\ -3 & -2\end{array}\right], P A P^{-1}=\left[\begin{array}{cc}x & -b(2 a-3 b)+a(3 a-2 b) \\ \times & \times\end{array}\right] \neq\left[\begin{array}{ll}\times & 1 \\ \times & \times\end{array}\right]$ because otherwise, $1=-b(2 a-3 b)+a(3 a-2 b)=3 a^{2}+3 b^{2}-4 a b$ will imply that $a=\frac{1}{3}\left(2 b \pm \sqrt{-5 b^{2}+3}\right) \notin \mathbb{Z}$, a contradiction.

For the second matrix, we see that $P A P^{-1}=\left[\begin{array}{cc}d(a+2 b)-c(2 a-b) & \times \\ \times & \times\end{array}\right] \neq$ $\left[\begin{array}{cc}0 & \times \\ \times & \times\end{array}\right]$ because $d a+2 d b-2 a c+b c=1+2 b c+2 d b-2 a c \neq 0$.

The same method works for the remaining matrices.

We next show that each of the polynomials in (II) corresponds to only two $\mathbb{Z}$-similar classes.

(A) $x^{2}+5$. Note that $\theta=\sqrt{-5}$ is a root of $x^{2}+5$. By Method I and Table 1 in Section 2, we see that the class number of $Q(\sqrt{-5})$ is 2 .

(B) $x^{2}-5$. We use Method II. We see that $a_{11}=-a_{22}$ and $p(A)=a_{11}^{2} \geq 0$. We divide our consideration into parts: 
(i) If $p(A)=0$, then Proposition 2.2 implies that $A$ is $\mathbb{Z}$-similar to the companion matrix.

(ii) If $p(A)>0$ and $\left|a_{11}\right|=1$, then $p(A)=a_{11}^{2}=1$ implies that $a_{12} a_{21}=$ $-p(A)-\operatorname{det}(A)=4$. We can list all the possible cases for $\mathrm{A}$, and check by Proposition 2.1 to conclude that $A \sim\left[\begin{array}{rr}1 & 2 \\ 2 & -1\end{array}\right]$ or $\left[\begin{array}{ll}0 & \times \\ \times & \times\end{array}\right]$ (which is $\mathbb{Z}$-similar to $C$ by Proposition 2.2).

(iii) If $p(A)>0$ and $\left|a_{11}\right|=2$, then $p(A)=a_{11}^{2}=4$ implies that $a_{12} a_{21}=$ $-p(A)-\operatorname{det}(A)=1$. We arrive at the same conclusion as (i).

(iv) If $p(A)>0$ and $\left|a_{11}\right| \geq 3$, then we use Lemma 5.2 and part $\mathrm{B}$ (vii) in the proof of Theorem 4.2 to reduce $A$ to a $\mathbb{Z}$-similar matrix in (i)-(iii).

(C) $x^{2} \pm 2 x+5$. By Lemma 2.7, we only need to consider $x^{2}-2 x+5$. Note that $a_{11}+a_{22}=2$ implies that $p(A)=-a_{11} a_{22} \geq-1$. We consider the following cases:

(i) If $p(A)=-1$, then $a_{11}=a_{22}=1$ and $a_{12} a_{21}=-p(A)-\operatorname{det}(A)=-4$. By checking the possible cases, $A$ is either $\mathbb{Z}$-similar to $\left[\begin{array}{rr}1 & 2 \\ -2 & 1\end{array}\right]$ or $\left[\begin{array}{ll}0 & \times \\ \times & \times\end{array}\right]$, which is $\mathbb{Z}$-similar to $C$ by Proposition 2.2.

(ii) $p(A)=0$. Then $A \sim C$ by Proposition 2.2 .

(iii) $p(A)>0$ and $\left|a_{11}\right|=1$. It is easy to show that $a_{11}=-1$, so that $a_{22}=3$ and $a_{12} a_{21}=-p(A)-\operatorname{det}(A)=-8 . A$ is of the form $\left[\begin{array}{rr}-1 & \mp 8 \\ \pm 1 & 3\end{array}\right],\left[\begin{array}{rr}-1 & \mp 4 \\ \pm 2 & 3\end{array}\right]$ (and their transposes). The first matrix is $\mathbb{Z}$-similar to $\left[\begin{array}{ll}0 & x \\ x & \times\end{array}\right]$ by Proposition 2.1. For the second one, we note that $\left[\begin{array}{rr}1 & -1 \\ 0 & 1\end{array}\right]\left[\begin{array}{ll}-1 & 4 \\ -2 & 3\end{array}\right]\left[\begin{array}{ll}1 & 1 \\ 0 & 1\end{array}\right]=\left[\begin{array}{rr}1 & 2 \\ -2 & 1\end{array}\right]$.

(iv) $p(A)>0$ and $\left|a_{11}\right|=2$. The same argument as above shows that $a_{11}=-2$, $a_{22}=4$ and $a_{12} a_{21}=-p(A)-\operatorname{det}(A)=-13$. Therefore Proposition 2.1 implies that $A \sim\left[\begin{array}{ll}0 & \times \\ \times & \times\end{array}\right]$, which is $\mathbb{Z}$-similar to $C$.

(v) $p(A)>0$ and $\left|a_{11}\right|=3$. Then $\left(a_{11}, a_{22}\right)=(3,-1),(-3,5)$ which implies that $a_{12} a_{21}=-p(A)-\operatorname{det}(A)=-8,-20$. For all the possible $A$, except the following two, they are quite easy to check that they are similar to $\left[\begin{array}{ll}0 & x \\ x & x\end{array}\right]$ by Proposition 2.1. The two less straightforward cases are $\left[\begin{array}{ll}3 & -4 \\ 2 & -1\end{array}\right] \sim\left[\begin{array}{rr}1 & 2 \\ -2 & 1\end{array}\right]$ (Proposition 2.1) and $\left[\begin{array}{rr}1 & -2 \\ 0 & 1\end{array}\right]\left[\begin{array}{rr}-3 & 10 \\ -2 & 5\end{array}\right]\left[\begin{array}{ll}1 & 2 \\ 0 & 1\end{array}\right]=\left[\begin{array}{rr}1 & 2 \\ -2 & 1\end{array}\right]$.

(vi) $p(A)>0$ and $\left|a_{11}\right|=4$. It is straightforward to check $\left(a_{11}, a_{22}\right)=(-4,6),(4$, -2). Hence $a_{12} a_{21}=-p(A)-\operatorname{det}(A)=-29,-13$. For all possible $A$ 's, it can be shown that $A \sim\left[\begin{array}{ll}0 & \times \\ \times & \times\end{array}\right]$.

(vii) $p(A)>0$ and $\left|a_{11}\right|>4$. We use Lemma 5.2 and proceed as in part $\mathrm{B}$ (vii) of the proof of Theorem 4.2 to reduce $A$ to a $\mathbb{Z}$-similar matrix in (i)-(vi).

\section{Characterization of 3-Digit Tiles in $\mathbb{R}^{2}$}

Let $A \in M_{n}(\mathbb{Z})$ be expanding with $|\operatorname{det}(A)|=q$ and let $D=\left\{d_{1}, \ldots, d_{q}\right\} \subseteq \mathbb{Z}^{n}$ be a $q$ digit set. We can always reduce to the case $0 \in D$ by a translation. Assume that $T(A, D)$ is a tile. Let $L$ be the lattice generated by $D, A D, \ldots, A^{n-1} D$. The quotient group 
$L / A(L)$ has $q$ distinct cosets; $D \subset L$ is called a complete set of coset representatives of $L / A(L)$ if

$$
L=\bigcup_{i=1}^{q}\left(d_{i}+A(L)\right) \quad \text { and } \quad\left(d_{i}+A(L)\right) \cap\left(d_{j}+A(L)\right)=\emptyset \quad \text { for } \quad i \neq j .
$$

For simplicity, we just say that $D$ is complete in $L / A(L)$; in this case $\mu(T(A, D))>0$ [B]. It is known that if the columns of a matrix $B \in M_{2}(\mathbb{Z})$ form a basis of $L$, then for $\tilde{D}=B^{-1} D$ and $\tilde{A}=B^{-1} A B \in M_{n}(\mathbb{Z}), \mathbb{Z}[\tilde{A}, \tilde{D}]=\mathbb{Z}^{n}$ with $0 \in \tilde{D}$ [LW3]. Here $\mathbb{Z}[\tilde{A}, \tilde{D}]$ is the lattice generated by $\tilde{D}, \tilde{A} \tilde{D}, \ldots, \tilde{A}^{n-1} \tilde{D}$. Note that $T(\tilde{A}, \tilde{D})=$ $B^{-1} T(A, D)$. We say that $D$ is a standard digit set (with respect to $A$ ) if $D$ is complete in $L / A(L)$; otherwise, $D$ is called a nonstandard digit set. Then $D$ is a standard digit set (with respect to $A$ ) is equivalent to $\tilde{D}$ is a standard digit set with respect to $\tilde{A}$, i.e., $\tilde{D}$ is complete in $\mathbb{Z}^{n} / \tilde{A}\left(\mathbb{Z}^{n}\right)$. It is clear that if $D$ is a standard digit set and if $D^{\prime}=a D$ for some $a \in \mathbb{Z} \backslash\{0\}$, then $D^{\prime}$ is also a standard digit set. Also note that if $D$ is complete in $\mathbb{Z}^{n} / A\left(\mathbb{Z}^{n}\right)$, then $D$ is standard. However, the converse is not true (see Remark 1 after Corollary 6.7)

In [LW3] Lagarias and Wang proved the following theorem.

Theorem 6.1. Let $A \in M_{n}(\mathbb{Z})$ be an expanding matrix such that $|\operatorname{det}(A)|=q$ is a prime, and suppose that

$$
q \mathbb{Z}^{n} \nsubseteq A^{2}\left(\mathbb{Z}^{n}\right) .
$$

If $D \subseteq \mathbb{Z}^{n}$ is a digit set with $\# D=q$, then $\mu(T(A, D))>0$ if and only if $D$ is a standard digit set.

Condition (6.1) seems to be artificial and they conjecture that the condition is redundant. In the following we give a thorough investigation of the cases where $|\operatorname{det}(A)|=3$ and (6.1) is not satisfied. The conclusion of the theorem still holds.

Proposition 6.2. Let $f(x)=x^{2}-a x \pm q$ with $a \in \mathbb{Z}, q \in \mathbb{N}$ is a prime. Let $C$ be the companion matrix of $f(x)$. Then $q \mathbb{Z}^{2} \subseteq C^{2}\left(\mathbb{Z}^{2}\right)$ if and only if $q \mid a$.

Proof. The companion matrix is of the form $C=\left[\begin{array}{cc}0 & 1 \\ \varepsilon q & a\end{array}\right]$ where $\varepsilon= \pm 1$. For $\left[\begin{array}{l}k \\ l\end{array}\right] \in \mathbb{Z}^{2}$, the solution of $q\left[\begin{array}{l}k \\ l\end{array}\right]=C^{2}\left[\begin{array}{l}x \\ y\end{array}\right]$ is of the form

$$
x=\varepsilon\left(k-q^{-1} a y\right), \quad y=\varepsilon(l-a k) .
$$

If $q \mid a$, then it is easy to see that $x$ and $y$ are integers so that the sufficiency follows. To prove the necessity, we assume that $q \backslash \backslash a$, then we pick $\left[\begin{array}{l}k \\ l\end{array}\right] \in \mathbb{Z}^{2}$ such that $l-a k=1$. The solution $\left[\begin{array}{l}x \\ y\end{array}\right] \notin \mathbb{Z}^{2}$ and $q \mathbb{Z}^{2} \nsubseteq C^{2}\left(\mathbb{Z}^{2}\right)$.

It follows that for $|\operatorname{det}(A)|=3$, condition (6.1) holds except for $f(x)=x^{2} \pm 3, x^{2} \pm$ $3 x+3$. In view of the classification in Section 3, we need to consider the corresponding 
companion matrices, and for $f(x)=x^{2}+3$ we have to consider one more $\mathbb{Z}$-similar class $\left[\begin{array}{rr}1 & 2 \\ -2 & -1\end{array}\right]$. First, we need a result modified from [LW3].

Proposition 6.3. Let $A \in M_{n}(\mathbb{Z})$ be an expanding matrix such that $|\operatorname{det}(A)|=q$ is a prime. Let $D$ be a $q$-digit set such that $0 \in D$. Then $\mu(T(A, D))>0$ if and only if for each $m \in \mathbb{Z}^{2} \backslash\{0\}$, there exists a nonnegative integer $k$ (depends on $m$ ) such that $\left\{q\left\langle A^{-k} d, m\right\rangle: d \in D\right\}$ is complete in $\mathbb{Z}_{q}$.

Proof. By Theorem 2.1(iii) of [LW3], $\mu(T(A, D))>0$ if and only if for each $m \in$ $\mathbb{Z}^{2} \backslash\{0\}$, there exists a nonnegative integer $k$ (depends on $m$ ) such that the function $\hat{h}_{k}(x):=(1 / q) \sum_{d \in D} \exp \left(2 \pi i\left\langle A^{-k} d, x\right\rangle\right)$ has $\hat{h}_{k}(m)=0$. It is equivalent to saying that the set $\left\{\exp \left(2 \pi i\left\langle A^{-k} d, m\right\rangle\right): d \in D\right\}$ consists of distinct $q$ th roots of unity for each $m \in \mathbb{Z}^{2} \backslash\{0\}$ (see the proof of Theorem 4.1 in [LW3]). This is, in turn, equivalent to $\left\{q\left\langle A^{-k} d, m\right\rangle: d \in D\right\}$ is complete in $\mathbb{Z}_{q}$ for each $m \in \mathbb{Z}^{2} \backslash\{0\}$.

Lemma 6.4. Let $A=\left[\begin{array}{rr}0 & 1 \\ \pm 3 & 0\end{array}\right]$ be the companion matrix of $f(x)=x^{2} \mp 3$. Then $A^{2}= \pm 3 I$, and for any $k \in \mathbb{N}, d=\left[\begin{array}{l}d_{1} \\ d_{2}\end{array}\right], m=\left[\begin{array}{l}m_{1} \\ m_{2}\end{array}\right]$,

$$
\left\langle A^{-k} d, m\right\rangle= \begin{cases}( \pm 3)^{-k^{\prime}}\left(d_{2} m_{1} \pm 3 d_{1} m_{2}\right) & \text { if } \quad k=2 k^{\prime}-1, \\ ( \pm 3)^{-k^{\prime}}\left(d_{1} m_{1}+d_{2} m_{2}\right) & \text { if } \quad k=2 k^{\prime} .\end{cases}
$$

By Proposition 6.3 and Lemma 6.4, we have

Lemma 6.5. Let $A=\left[\begin{array}{rr}0 & 1 \\ \pm 3 & 0\end{array}\right], D=\left\{0, d, d^{\prime}\right\} \subseteq \mathbb{Z}^{2}$. For $m \in \mathbb{Z}^{2} \backslash\{0\}$, we let

$$
\begin{aligned}
\mathcal{O}(D, m) & =\left\{0, d_{2} m_{1} \pm 3 d_{1} m_{2}, d_{2}^{\prime} m_{1} \pm 3 d_{1}^{\prime} m_{2}\right\}, \\
\mathcal{E}(D, m) & =\left\{0, d_{1} m_{1}+d_{2} m_{2}, d_{1}^{\prime} m_{1}+d_{2}^{\prime} m_{2}\right\} .
\end{aligned}
$$

Then $\mu(T(A, D))>0$ if and only if for each $m \in \mathbb{Z}^{2} \backslash\{0\}$, there exists a nonnegative integer $k$ such that $3^{-k} \mathcal{O}(D, m)$ or $3^{-k} \mathcal{E}(D, m)$ is complete in $\mathbb{Z}_{3}$.

For $D=\left\{0, d, d^{\prime}\right\}=\left\{0,\left[\begin{array}{l}d_{1} \\ d_{2}\end{array}\right],\left[\begin{array}{l}d_{1}^{\prime} \\ d_{2}^{\prime}\end{array}\right]\right\}$, we let $D_{1}=\left\{0, d_{1}, d_{1}^{\prime}\right\}, D_{2}=\left\{0, d_{2}, d_{2}^{\prime}\right\}$ and let $k=\min \left\{k_{1}, k_{2}\right\}$ where $k_{1}$ and $k_{2}$ are the largest powers of the factor 3 for the sets $D_{1}$ and $D_{2}$, respectively.

Theorem 6.6. Let $A=\left[\begin{array}{rr}0 & 1 \\ \pm 3 & 0\end{array}\right]$ and let $D$ be the digit set as above. Then $\mu(T(A, D))$ $>0$ if and only if either one of the following holds:

(i) $k=k_{1}<k_{2}$ and $3^{-k} D_{1}$ is complete in $\mathbb{Z}_{3}$,

(ii) $k=k_{2} \leq k_{1}$ and $3^{-k} D_{2}$ is complete in $\mathbb{Z}_{3}$.

Proof. Without loss of generality, we assume that $k=0$. For $\mu(T(A, D))>0$, we let $m=\left[\begin{array}{l}1 \\ 0\end{array}\right]$, then by Lemma 6.5 , for some $k$, either $3^{-k} D_{1}$ or $3^{-k} D_{2}$ is complete in 
$\mathbb{Z}_{3}$. For case (i) with $0=k_{1}<k_{2}$, if $D_{1}$ is not complete in $\mathbb{Z}_{3}$, we consider $m=\left[\begin{array}{l}3 \\ 1\end{array}\right]$, then

$$
\begin{aligned}
& \mathcal{O}(D, m)=3\left\{0, d_{2} \pm d_{1}, d_{2}^{\prime} \pm d_{1}^{\prime}\right\}, \\
& \mathcal{E}(D, m)=3\left\{0, d_{1}+\bar{d}_{2}, d_{1}^{\prime}+\bar{d}_{2}^{\prime}\right\},
\end{aligned}
$$

where $\bar{d}_{2}=\frac{1}{3} d_{2}, \bar{d}_{2}^{\prime}=\frac{1}{3} d_{2}^{\prime}$ are integers. It is easy to see that

$$
\begin{aligned}
& 3^{-1} \mathcal{O}(D, m) \equiv\left\{0, \pm d_{1}, \pm d_{1}^{\prime}\right\} \quad(\bmod 3),
\end{aligned}
$$

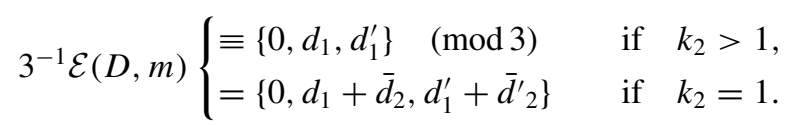

In all cases they are not complete in $\mathbb{Z}_{3}$ (for the case $k_{2}=1$, we use $\left\{0, \bar{d}_{2}, \bar{d}^{\prime}{ }_{2}\right\}$ is complete in $\mathbb{Z}_{3}$ ), hence a contradiction and (i) follows.

In case (ii) if $0=k_{2} \leq k_{1}$, and if $D_{2}$ is not complete in $\mathbb{Z}_{3}$, then we consider $m=\left[\begin{array}{l}1 \\ 1\end{array}\right]$, we see that

$$
\begin{aligned}
& \mathcal{O}(D, m)=\left\{0, d_{2} \pm 3 d_{1}, d_{2}^{\prime} \pm 3 d_{1}^{\prime}\right\}, \\
& \mathcal{E}(D, m)=\left\{0, d_{1}+d_{2}, d_{1}^{\prime}+d_{2}^{\prime}\right\} .
\end{aligned}
$$

The same argument shows that neither set is complete in $\mathbb{Z}_{3}$. This a contradiction.

To prove the sufficiency, we write $m_{1}=3^{s_{1}}\left(u_{1}+3 r_{1}\right), m_{2}=3^{s_{2}}\left(u_{2}+3 r_{2}\right)$ and assume without loss of generality that $s_{1}=0$ or $s_{2}=0$. If $m_{1}=0\left(m_{2}=0\right)$, we assume that $s_{1} \neq 0\left(s_{2} \neq 0\right)$. Now for case (i) if $s_{1}=0$, we see that $\mathcal{E}(D, m)$ is complete in $\mathbb{Z}_{3}$; if $s_{1} \neq 0$ and $s_{2}=0$, then the same conclusion holds for $3^{-1} \mathcal{O}(D, m)$. In case (ii) $\mathcal{O}(D, m)$ or $\mathcal{E}(D, m)$ are complete in $\mathbb{Z}_{3}$ according to the two cases $s_{1}=0$ or $s_{1} \neq 0$, $s_{2}=0$.

Corollary 6.7. Let $A$ and $D$ be as in Theorem 6.6. Then $\mu(T(A, D))>0$ if and only if $D$ is a standard digit set.

Proof. We assume that $k=0$ in the above theorem. We will show that either (i) or (ii) implies that $D$ is standard. Observe that $A\left(\mathbb{Z}^{2}\right)=\left\{\left[\begin{array}{c}n \\ 3 m\end{array}\right]:\left[\begin{array}{c}m \\ n\end{array}\right] \in \mathbb{Z}^{2}\right\}$ and $\mathbb{Z}^{2} / A\left(\mathbb{Z}^{2}\right)$ has equivalence classes $\left\{\left[\begin{array}{l}0 \\ 0\end{array}\right],\left[\begin{array}{l}0 \\ 1\end{array}\right],\left[\begin{array}{l}0 \\ 2\end{array}\right]\right\}$.

In case (ii) of Theorem 6.6, $D_{2}$ is complete in $\mathbb{Z}_{3}$, hence $D$ is complete in $\mathbb{Z}^{2} / A\left(\mathbb{Z}^{2}\right)$ and is a standard digit set.

In case (i) of Theorem 6.6, $D_{1}$ is complete. We will construct an invertible matrix $B$ as in the definition of the standard digit set. Observe that $A d=\left[\begin{array}{c}\times \\ 3 d_{1}\end{array}\right], A d^{\prime}=\left[\begin{array}{c}\times \\ 3 d_{1}^{\prime}\end{array}\right]$. Let $B$ be a matrix such that the columns of it are a basis of the lattice $L$ defined as in the beginning of this section. Then $B$ is of the form

$$
B=\left[\begin{array}{cc}
\times & \times \\
3 r & 3 s
\end{array}\right]
$$


Let $\left\{0, \tilde{d}, \tilde{d}^{\prime}\right\}=B^{-1} D \in \mathbb{Z}^{2}$ and $\tilde{A}=B^{-1} A B \in M_{n}(\mathbb{Z})$, we claim that $\tilde{D}$ is complete in $\mathbb{Z}^{2} / \tilde{A}\left(\mathbb{Z}^{2}\right)$, hence $D$ is a standard digit set. We observe that $\tilde{d} \in \mathbb{Z}^{2} / \tilde{A}\left(\mathbb{Z}^{2}\right)$ if and only if $B^{-1} d=B^{-1} A B z$ for some $z=\left[\begin{array}{l}z_{1} \\ z_{2}\end{array}\right] \in \mathbb{Z}^{2}$. This implies that

$$
\left[\begin{array}{c}
d_{1} \\
\times
\end{array}\right]=A B\left[\begin{array}{l}
z_{1} \\
z_{2}
\end{array}\right]=\left[\begin{array}{c}
3\left(r z_{1}+s z_{2}\right) \\
\times
\end{array}\right],
$$

and $d_{1}$ has factor 3 , an impossibility since $D_{1}$ is complete in $Z_{3}$. We hence conclude that $\tilde{d} \notin \mathbb{Z}^{2} / \tilde{A}\left(\mathbb{Z}^{2}\right)$. Similarly we make the same conclusion for $\tilde{d}^{\prime}$ and $\tilde{d}-\tilde{d}^{\prime}$ and the claim follows.

Remark 1. Corollary 6.7 shows that the fact that $D$ is complete in $L / A(L)$ does not imply that $D$ is complete in $\mathbb{Z}^{2} / A\left(\mathbb{Z}^{2}\right)$.

Remark 2. In Theorem 6.6 and Corollary 6.7, $A$ and $D$ can be replaced by $A=$ $\left[\begin{array}{rr}0 & 1 \\ \pm q & 0\end{array}\right], D=\left\{0, d_{1}, \ldots, d_{q-1}\right\} \subseteq \mathbb{Z}^{2}$, where $q$ is a prime. In this case, $D_{1}$ and $D_{2}$ consist of first and second entries of the digits of $D$, respectively.

Next, we consider the $\mathbb{Z}$-similar class $A=\left[\begin{array}{rr}1 & 2 \\ -2 & -1\end{array}\right]$ corresponding to the characteristic polynomial $f(x)=x^{2}+3$. By using the same observation as Lemma 6.5, we have

Lemma 6.8. Let $A=\left[\begin{array}{rr}1 & 2 \\ -2 & -1\end{array}\right], D=\left\{0, d, d^{\prime}\right\} \subseteq \mathbb{Z}^{2}$. Then $A^{2}=-3 I$ and for $m \in \mathbb{Z}^{2} \backslash\{0\}$, we let

$$
\begin{aligned}
\mathcal{O}(D, m) & =\left\{0,\left(m_{1}-2 m_{2}\right) d_{1}+\left(2 m_{1}-m_{2}\right) d_{2},\left(m_{1}-2 m_{2}\right) d_{1}^{\prime}+\left(2 m_{1}-m_{2}\right) d_{2}^{\prime}\right\}, \\
\mathcal{E}(D, m) & =\left\{0, m_{1} d_{1}+m_{2} d_{2}, m_{1} d_{1}^{\prime}+m_{2} d_{2}^{\prime}\right\} .
\end{aligned}
$$

Then $\mu(T(A, D))>0$ if and only if for each $m \in \mathbb{Z}^{2} \backslash\{0\}$, there exists a nonnegative integer $k$ such that $3^{-k} \mathcal{O}(D, m)$ or $3^{-k} \mathcal{E}(D, m)$ is complete in $\mathbb{Z}_{3}$.

We use the same notation as in the last theorem. For the digit set $D$, we let $D_{1}=$ $\left\{0, d_{1}, d_{1}^{\prime}\right\}, D_{2}=\left\{0, d_{2}, d_{2}^{\prime}\right\}$, and let $k=\min \left\{k_{1}, k_{2}\right\}$ where $k_{1}$ and $k_{2}$ are the largest powers of the factor 3 for the sets $D_{1}$ and $D_{2}$, respectively.

Theorem 6.9. Let $A, D=\left\{0, d, d^{\prime}\right\}$ be as above, then $\mu(T(A, D))>0$ if and only if there exists an integer $k \geq 0$ such that one of the following (nonexclusive) cases holds:

(i) $k=k_{1}<k_{2}$ and $3^{-k} D_{1}$ is complete in $\mathbb{Z}_{3}$,

(ii) $k=k_{2}<k_{1}$ and $3^{-k} D_{2}$ is complete in $\mathbb{Z}_{3}$,

(iii) $k=k_{1}=k_{2}$ and either one of the following holds:

(a) $3^{-k} D \equiv\left\{\left[\begin{array}{l}0 \\ 0\end{array}\right],\left[\begin{array}{l}0 \\ j\end{array}\right],\left[\begin{array}{l}i \\ j\end{array}\right]: i \neq j, i, j=1,2\right\}(\bmod 3)$ or $3^{-k} D \equiv\left\{\left[\begin{array}{l}0 \\ 0\end{array}\right],\left[\begin{array}{l}j \\ i\end{array}\right],\left[\begin{array}{l}j \\ 0\end{array}\right]: i \neq j, i, j=1,2\right\}(\bmod 3)$; 
(b) $3^{-k} D \equiv\left\{\left[\begin{array}{l}0 \\ 0\end{array}\right],\left[\begin{array}{l}i \\ 0\end{array}\right],\left[\begin{array}{l}0 \\ j\end{array}\right]: i=j, i, j=1,2\right\}(\bmod 3)$;

(c) both $3^{-k} D_{1}$ and $3^{-k} D_{2}$ are complete in $\mathbb{Z}_{3}$.

Proof. Without loss of generality we assume that $k=0$. Suppose $\mu(T(A, D))>0$. If $0=k_{1}<k_{2}$, we take $m=\left[\begin{array}{l}1 \\ 0\end{array}\right]$, then

$$
\mathcal{O}(D, m)=\left\{0, d_{1}+2 d_{2}, d_{1}^{\prime}+2 d_{2}^{\prime}\right\}, \quad \mathcal{E}(D, m)=\left\{0, d_{1}, d_{1}^{\prime}\right\}
$$

It follows that $\mathcal{O}(D, m), \mathcal{E}(D, m)$ are complete in $\mathbb{Z}_{3}$ if and only if one of them is. By the above lemma, we see that (i) holds. If $0=k_{2}<k_{1}$, we use $m=\left[\begin{array}{l}0 \\ 1\end{array}\right]$ and consider

$$
\mathcal{O}(D, m)=\left\{0,-2 d_{1}-d_{2},-2 d_{1}^{\prime}-d_{2}^{\prime}\right\}, \quad \mathcal{E}(D, m)=\left\{0, d_{2}, d_{2}^{\prime}\right\}
$$

then the same argument as above shows that (ii) holds. If $0=k_{1}=k_{2}$ and only one of $D_{1}$ and $D_{2}$ is complete in $\mathbb{Z}_{3}$, we consider $m=\left[\begin{array}{l}1 \\ 1\end{array}\right]$. Then one can see that

$$
\mathcal{O}(D, m)=\left\{0,-d_{1}+d_{2},-d_{1}^{\prime}+d_{2}^{\prime}\right\}, \quad \mathcal{E}(D, m)=\left\{0, d_{1}+d_{2}, d_{1}^{\prime}+d_{2}^{\prime}\right\}
$$

are not complete in $\mathbb{Z}_{3}$. If $0=k_{1}=k_{2}$ and if none of $D_{1}$ and $D_{2}$ is complete in $\mathbb{Z}_{3}$, then we use $m=\left[\begin{array}{l}1 \\ 0\end{array}\right]$ and $\left[\begin{array}{l}0 \\ 1\end{array}\right]$ and Lemma 6.8 to conclude that both

$$
\left\{0, d_{1}+2 d_{2}, d_{1}^{\prime}+2 d_{2}^{\prime}\right\}, \quad\left\{0,-2 d_{1}-d_{2},-2 d_{1}^{\prime}-d_{2}^{\prime}\right\}
$$

are complete in $\mathbb{Z}_{3}$. It is now direct to check that (iii) holds.

To prove the sufficiency, we write $m_{i}=3^{s_{i}}\left(u_{i}+3 r_{i}\right), i=1,2$, and assume without loss of generality that $s_{1}$ or $s_{2}=0$. If $m_{i}=0, i=1,2$, then we also assume $s_{i}$ is arbitrarily large.

For case (i), if $s_{1}=0$, then $\mathcal{E}(D, m)$ is complete; if $s_{1} \neq 0, s_{2}=0$, then $\mathcal{O}(D, m) \equiv$ $\left\{0,-2 u_{2} d_{1},-2 u_{2} d_{1}^{\prime}\right\} \equiv\left\{0, d_{1}, d_{1}^{\prime}\right\}(\bmod 3)$ and is complete in $\mathbb{Z}_{3}$ by assumption.

For case (ii), the same technique will work.

For case (iii)(a), we give a proof for $d_{1} \equiv 0(\bmod 3)$ since the other cases can be handled similarly. If $0=s_{2}<s_{1}$, then $\mathcal{O}(D, m) \equiv\left\{0, u_{2} 2 j, u_{2}(i+2 j)\right\}$ is complete in $\mathbb{Z}_{3}$; if $0=s_{1}<s_{2}$, then $\mathcal{O}(D, m)=\left\{0,2 u_{1} j, u_{1}(i+2 j)\right\}$ is complete in $\mathbb{Z}_{3}$; if $0=s_{1}=s_{2}$, for $u_{1}=u_{2}=u$, then $\mathcal{O} \equiv\{0, u j, u(-i+j)\}$ is complete in $\mathbb{Z}_{3}$, and for $u_{1} \neq u_{2}, u_{1}, u_{2}=1,2$, then $\mathcal{E} \equiv\left\{0, u_{2} j, u_{1} i+u_{2} j\right\}$ is complete in $\mathbb{Z}_{3}$.

For case (iii)(b), if $0=s_{1}<s_{2}$, then $\mathcal{O}(D, m) \equiv\left\{0, u_{1} i, 2 u_{1} j\right\}$ is complete in $\mathbb{Z}_{3}$; if $0=s_{2}<s_{1}$, then $\mathcal{O}(D, m) \equiv\left\{0,-2 u_{2} i,-u_{2} j\right\}$ is complete in $\mathbb{Z}_{3}$; if $0=s_{2}=s_{1}$, then for $u=u_{1}=u_{2}, \mathcal{O}(D, m) \equiv\{0,-u i, u j\}$ is complete in $\mathbb{Z}_{3}$, and for $u_{1} \neq u_{2}$, $\mathcal{E}(D, m) \equiv\left\{0, u_{1} i, u_{2} j\right\}$ is complete in $\mathbb{Z}_{3}$.

For case (iii)(c), if $0=s_{1}<s_{2}$ or $0=s_{2}<s_{1}$, then $\mathcal{E}(D, m)$ is complete in $\mathbb{Z}_{3}$. Suppose that $0=s_{2}=s_{1}$. We let $\left(0, d_{1}, d_{2}\right)=(0, i, j),\left(0, d_{1}^{\prime}, d_{2}^{\prime}\right)=\left(0, i^{\prime}, j^{\prime}\right)$, 
$i, j, i^{\prime}, j^{\prime}=1,2$. For $u_{1}=u_{2}$, we can check that if $i \neq j$, then $\mathcal{O}(D, m)$ is complete in $\mathbb{Z}_{3}$; if $i=j$, then $\mathcal{E}(D, m)$ is complete in $\mathbb{Z}_{3}$. For $u_{1} \neq u_{2}, \mathcal{E}(D, m)$ or $3^{-1} \mathcal{E}(D, m)$ is complete in $\mathbb{Z}_{3}$.

Corollary 6.10. Let $A$ and $D$ be as in Theorem 6.9. Then $\mu(T(A, D))>0$ if and only if $D$ is a standard digit set.

Proof. By a direct calculation it is easy to see that $\left[\begin{array}{l}x \\ y\end{array}\right] \in A \mathbb{Z}^{2}$ if and only if $3 \mid(x-y)$. Using this observation, it follows that except for one case (assuming that $k=0$ ) the digit sets $D$ in Theorem 6.9 are complete in $\mathbb{Z}^{2} / A\left(\mathbb{Z}^{2}\right)$. The exceptional case is

$$
d=\left[\begin{array}{l}
1+3 r_{1} \\
1+3 r_{2}
\end{array}\right], \quad d^{\prime}=\left[\begin{array}{l}
2+3 r_{1}^{\prime} \\
2+3 r_{2}^{\prime}
\end{array}\right]
$$

(assuming the $3^{k}$ factor is 1 ). Note that

$$
A d=\left[\begin{array}{c}
3+3\left(r_{1}+2 r_{2}\right) \\
-\left(3+3\left(2 r_{1}+r_{2}\right)\right)
\end{array}\right], \quad A d^{\prime}=\left[\begin{array}{c}
6+3\left(r_{1}^{\prime}+2 r_{2}^{\prime}\right) \\
-\left(6+3\left(2 r_{1}^{\prime}+r_{2}^{\prime}\right)\right)
\end{array}\right] .
$$

Let $B=\left[b, b^{\prime}\right]$ be a basis for the lattice $\mathbb{Z}[A, D]$. Then $b=\alpha_{1} d+\alpha_{2} d^{\prime}+\alpha_{3} A d+\alpha_{4} A d^{\prime}$, $b^{\prime}=\beta_{1} d+\beta_{2} d^{\prime}+\beta_{3} A d+\beta_{4} A d^{\prime}$ for some $\alpha_{i}, \beta_{i} \in \mathbb{Z}, i=1,2,3,4$. Then

$$
B=\left[\begin{array}{ll}
\alpha_{1}+2 \alpha_{2}+3 r & \beta_{1}+2 \beta_{2}+3 r^{\prime} \\
\alpha_{1}+2 \alpha_{2}+3 s & \beta_{1}+2 \beta_{2}+3 s^{\prime}
\end{array}\right]
$$

for some $r, r^{\prime}, s, s^{\prime} \in \mathbb{Z}$. Let $\tilde{D}=B^{-1} D \in \mathbb{Z}^{2}$ and $\tilde{A}=B^{-1} A B \in M_{2}(\mathbb{Z})$. We claim that $\tilde{D}$ is complete in $\mathbb{Z}^{2} / \tilde{A}\left(\mathbb{Z}^{2}\right)$ and hence $D$ is a standard digit set. We observe that $\tilde{d} \in \tilde{A}\left(\mathbb{Z}^{2}\right)$ if and only if there exists $z=\left[\begin{array}{l}z_{1} \\ z_{2}\end{array}\right] \in \mathbb{Z}$ such that $\tilde{d}=\tilde{A} z$. It follows that

$$
d=A B z=3\left[\begin{array}{c}
{\left[\left(\alpha_{1}+2 \alpha_{2}\right)+(r+2 s)\right] z_{1}+\left[\left(\beta_{1}+2 \beta_{2}\right)+\left(r^{\prime}+2 s^{\prime}\right)\right] z_{2}} \\
-\left[\left(\alpha_{1}+2 \alpha_{2}\right)+(2 r+s)\right] z_{1}-\left[\left(\beta_{1}+2 \beta_{2}\right)+\left(2 r^{\prime}+s^{\prime}\right)\right] z_{2}
\end{array}\right]
$$

which is impossible. Hence $\tilde{d} \notin \tilde{A}\left(\mathbb{Z}^{2}\right)$. The same argument holds for $\tilde{d}^{\prime}$ and $\tilde{d}-\tilde{d}^{\prime}$.

Finally, we consider the unique $\mathbb{Z}$-similar class $A=\left[\begin{array}{rr}0 & 1 \\ -3 & -3\end{array}\right]$ which has characteristic polynomial $f(x)=x^{2}+3 x+3$ (we do not need to consider $f(x)=x^{2}-3 x+3$ because of the remark in Section 2). Note that

$$
A^{-(6 k-5)}=(-1)^{k+1} 3^{-(3 k-2)}\left[\begin{array}{rr}
-3 & -1 \\
3 & 0
\end{array}\right]:=(-1)^{k+1} 3^{-(3 k-2)} A_{1},
$$




$$
\begin{aligned}
A^{-(6 k-4)} & =(-1)^{k+1} 3^{-(3 k-2)}\left[\begin{array}{rr}
2 & 1 \\
-3 & -1
\end{array}\right]:=(-1)^{k+1} 3^{-(3 k-2)} A_{2}, \\
A^{-(6 k-3)} & =(-1)^{k+1} 3^{-(3 k-1)}\left[\begin{array}{rr}
-3 & -2 \\
6 & 3
\end{array}\right]:=(-1)^{k+1} 3^{-(3 k-1)} A_{3}, \\
A^{-(6 k-2)} & =(-1)^{k+1} 3^{-(3 k-1)}\left[\begin{array}{rr}
1 & 1 \\
-3 & -2
\end{array}\right]:=(-1)^{k+1} 3^{-(3 k-1)} A_{4}, \\
A^{-(6 k-1)} & =(-1)^{k+1} 3^{-3 k}\left[\begin{array}{rr}
0 & -1 \\
3 & 3
\end{array}\right]:=(-1)^{k+1} 3^{-3 k} A_{5}, \\
A^{-6 k} & =(-1)^{k} 3^{-3 k}\left[\begin{array}{rr}
1 & 0 \\
0 & 1
\end{array}\right]:=(-1)^{k+1} 3^{-3 k} A_{6} .
\end{aligned}
$$

Let $\left\{\left\langle A_{i} d, m\right\rangle: d \in D\right\}=\mathcal{S}_{i}(D, m), i=1, \ldots, 6$. Then

$$
\begin{aligned}
\mathcal{S}_{1}(D, m)= & \left\{0,3\left(m_{2}-m_{1}\right) d_{1}-m_{1} d_{2}, 3\left(m_{2}-m_{1}\right) d_{1}^{\prime}-m_{1} d_{2}^{\prime}\right\}, \\
\mathcal{S}_{2}(D, m)= & \left\{0,\left(2 m_{1}-3 m_{2}\right) d_{1}+\left(m_{1}-m_{2}\right) d_{2},\left(2 m_{1}-3 m_{2}\right) d_{1}^{\prime}+\left(m_{1}-m_{2}\right) d_{2}^{\prime}\right\}, \\
\mathcal{S}_{3}(D, m)=\left\{0,\left(6 m_{2}-3 m_{1}\right) d_{1}+\left(-2 m_{1}+3 m_{2}\right) d_{2},\left(6 m_{2}-3 m_{1}\right) d_{1}^{\prime}\right. & \left.+\left(-2 m_{1}+3 m_{2}\right) d_{2}^{\prime}\right\}, \\
\mathcal{S}_{4}(D, m)= & \left\{0,\left(m_{1}-3 m_{2}\right) d_{1}+\left(m_{1}-2 m_{2}\right) d_{2},\left(m_{1}-3 m_{2}\right) d_{1}^{\prime}+\left(m_{1}-2 m_{2}\right) d_{2}^{\prime}\right\}, \\
\mathcal{S}_{5}(D, m)= & \left\{0,3 m_{2} d_{1}+\left(3 m_{2}-m_{1}\right) d_{2}, 3 m_{2} d_{1}^{\prime}+\left(3 m_{2}-m_{1}\right) d_{2}^{\prime}\right\}, \\
\mathcal{S}_{6}(D, m)= & \left\{0, m_{1} d_{1}+m_{2} d_{2}, m_{1} d_{1}^{\prime}+m_{2} d_{2}^{\prime}\right\} .
\end{aligned}
$$

By considering the set $\left\{3\left\langle A^{-k} d, m\right\rangle: d \in D\right\}$ as in Proposition 6.3, we have the following criterion for $T(A, D)$ to be a tile.

Lemma 6.11. Let $A$ be as above and let $D=\left\{0, d, d^{\prime}\right\} \in \mathbb{Z}^{2}$. Then $\mu(T(A, D))>0$ if and only if for each $m \in \mathbb{Z}^{2} \backslash\{0\}$, there exists a nonnegative integer $k$ such that either one of the sets $3^{k} \mathcal{S}_{i}(D, m), 1 \leq i \leq 6$, is complete in $\mathbb{Z}_{3}$.

Theorem 6.12. Let $A=\left[\begin{array}{rr}0 & 1 \\ -3 & -3\end{array}\right]$ and let $D=\left\{\left[\begin{array}{l}0 \\ 0\end{array}\right],\left[\begin{array}{l}d_{1} \\ d_{2}\end{array}\right],\left[\begin{array}{l}d_{1}^{\prime} \\ d_{2}^{\prime}\end{array}\right]\right\}$. Then $\mu(T(A, D))>$ 0 if and only if there exists $k$ such that either one of the following holds:

(i) $k=k_{1}<k_{2}$, then $3^{-k} D_{1}$ is complete in $\mathbb{Z}_{3}$,

(ii) $k=k_{2} \leq k_{1}$ : if $3^{-k} D_{2} \neq\{0,0,0\}$, then it is complete in $\mathbb{Z}_{3}$; otherwise $D_{1}$ is complete in $\mathbb{Z}_{3}$.

Moreover either (i) or (ii) implies that D is a standard digit set with respect to A. 
Proof. Without loss of generality, we assume that $k=0$. We first prove the necessity. Suppose that $\mu(T(A, D))>0$ and we want to show:

(1) Either $D_{1}$ or $D_{2}$ is complete in $\mathbb{Z}_{3}$.

(2) If $k=k_{1}=k_{2}=0$, then if $D_{2} \neq\{0,0,0\}$, it is complete in $\mathbb{Z}_{3}$, otherwise $D_{1}$ is complete in $\mathbb{Z}_{3}$

For $m=\left[\begin{array}{l}1 \\ 1\end{array}\right]$, a direct calculation yields

$$
\begin{array}{ll}
S_{1}(D, m) \equiv\left\{0,-d_{2},-d_{2}^{\prime}\right\} & (\bmod 3), \\
S_{2}(D, m) \equiv\left\{0,-d_{1},-d_{1}^{\prime}\right\} & (\bmod 3) .
\end{array}
$$

Lemma 6.11 implies that one of the above two sets must be complete in $\mathbb{Z}_{3}$ and (i) follows. To prove (ii) assume that $k=k_{1}=k_{2}=0$. For $m=\left[\begin{array}{l}2 \\ 1\end{array}\right]$, Lemma 6.11 implies that one of the above sets must be complete in $\mathbb{Z}_{3}$ :

$$
\begin{aligned}
& S_{5}(D, m) \equiv\left\{0, d_{2}, d_{2}^{\prime}\right\} \quad(\bmod 3) \\
& S_{2}(D, m) \equiv\left\{0, d_{1}+d_{2}, d_{1}^{\prime}+d_{2}^{\prime}\right\} \quad(\bmod 3)
\end{aligned}
$$

Suppose that $D_{2}=\{0,0,0\}$, then by (1), $D_{1}$ is complete in $\mathbb{Z}_{3}$. If $D_{2} \neq\{0,0,0\}$, then it must be complete in $\mathbb{Z}_{3}$. For otherwise, $D_{1}$ must be complete in $\mathbb{Z}_{3}$. However, in this case, none of the sets in (6.2) and (6.3) is complete in $\mathbb{Z}_{3}$, which is a contradiction. The necessity follows from (1) and (2).

To prove the sufficiency, it is suffice to show first that the digits in (i) and (ii) are standard. The proof is similar to the proof of Corrollary 6.7 and hence we omit it here.

\section{Self-Similarity}

A transformation $S: \mathbb{R}^{n} \rightarrow \mathbb{R}^{n}$ is called a similitude if there is a constant $\alpha>0$ such that $|S(x)-S(y)|=\alpha|x-y|$ for all $x, y \in \mathbb{R}^{n}$. In this case, $S$ is of the form $S x=M x+b$ where $M$ is a self-similar matrix, i.e., $|M x|=\alpha|x|$ for all $x \in \mathbb{R}^{n}$. The following proposition follows directly from the definition.

Proposition 7.1. $\quad M=\left[\begin{array}{ll}a & b \\ c & d\end{array}\right] \neq 0$ is a a self-similar matrix if and only if $c=\mp b$ and $d= \pm a$.

Corollary 7.2. If $A$ is $\mathbb{Z}$-similar to a self-similar matrix, then the trace of $A$ is $2 k$, $k \in \mathbb{Z}$.

Remark. For $A=\left[\begin{array}{ll}0 & 1 \\ u & v\end{array}\right]$ to be $\mathbb{Z}$-similar to $M=\left[\begin{array}{cc}m & n \\ n & -m\end{array}\right]$, we must have $v=0$. An example is $A=\left[\begin{array}{cc}0 & 1 \\ k^{2}+1 & 0\end{array}\right], k \neq 0$. Then $A \sim\left[\begin{array}{cc}k & 1 \\ 1 & -k\end{array}\right]$ using $P=\left[\begin{array}{cc}1 & 0 \\ -k & 1\end{array}\right]$. 
Corollary 7.3. Let $A=\left[\begin{array}{ll}0 & 1 \\ u & v\end{array}\right]$ and $v \neq 0$. Suppose $A$ is $\mathbb{Z}$-similar to a self-similar matrix $M$, then $u=-\left(k^{2}+1\right), v=2 k$ for some $k \in \mathbb{Z} \backslash\{0\}$. In this case, $M=\left[\begin{array}{cc}k & 1 \\ -1 & k\end{array}\right]$. Conversely, if $u, v$ in $A$ are given as above, then $A$ is $\mathbb{Z}$-similar to an $M$ of the above form.

Proof. Let $P=\left[\begin{array}{ll}a & b \\ c & d\end{array}\right]$ denote a unimodular matrix. We consider $A=P^{-1} M P$. $v \neq 0$ implies that $M$ is of the form $M=\left[\begin{array}{cc}m & n \\ -n & m\end{array}\right]$. By simplifying the expression, we have

$$
\begin{aligned}
& 0=a(m d+n b)+c(n d-m b), \\
& 1=\left(b^{2}+d^{2}\right) n, \\
& u=-\left(a^{2}+c^{2}\right) n, \\
& v=-2(a b+c d) n \quad \text { (using the first equation). }
\end{aligned}
$$

The equations imply that $n=1, b=0, d= \pm 1$ or $n=1, b= \pm 1, d=0$. This together with $a d-b c= \pm 1$ implies that $u=-\left(k^{2}+1\right), v=2 k$ with $k \neq 0$.

For the converse, we take $P=\left[\begin{array}{cc}1 & 0 \\ -k & 1\end{array}\right]$ and $M=\left[\begin{array}{cc}k & 1 \\ -1 & k\end{array}\right]$. Then $P^{-1} M P=A$.

We can use the above results to check if the expanding matrices in Sections 3-5 are $\mathbb{Z}$-similar to self-similar matrices. We first note that $A \in M_{2}(\mathbb{Z})$ with $A \sim M=$ $\left[\begin{array}{cc}m & n \\ \mp n & \pm m\end{array}\right]$ implies that the characteristic polynomial must have an even middle coefficient (Corollary 7.2). By the remark after Corollary 7.2, we see that for $A \in M_{2}(\mathbb{Z})$ with $\operatorname{det} A<0$ to have $A \sim M$, the characteristic polynomial should be of the form $x^{2}-q$. By the proof of Corollary 7.3, if $x^{2}+q$ is the characteristic polynomial of $A \in M_{2}(\mathbb{Z})$ with $\operatorname{det} A>0$, then the companion matrix $C \nsucc M$.

$|\operatorname{det}(A)|=2$. The $\mathbb{Z}$-similar classes are determined by the six companion matrices [LW1]. By Corollary 7.2, we need to consider the expanding polynomials $x^{2}-2, x^{2} \pm$ $2 x+2$. By the remark and Corollary 7.3, the companion matrices of $x^{2}-2, x^{2} \pm 2 x+2$ are $\mathbb{Z}$-similar to a self-similar matrix.

$|\operatorname{det}(A)|=3$. Since $m^{2}+n^{2}=3$ has no integer solutions, none of such $A$ will be $\mathbb{Z}$-similar to a self-similar matrix.

$|\operatorname{det}(A)|=4$. We observe that $m^{2}+n^{2}=4$ if and only if $m=0, n= \pm 2$ or $m= \pm 2, n=0$. Hence trace $(A)=0, \pm 4$. Therefore, we need to consider the expanding polynomials: $x^{2} \pm 4, x^{2} \pm 4 x+4$. For $x^{2}+4$, we have $M=\left[\begin{array}{rr}0 & 2 \\ -2 & 0\end{array}\right]$ and $C \nsucc M$ by Corollary 7.3. For $x^{2}-4$, there are three $\mathbb{Z}$-similar classes and two of them are $\mathbb{Z}$-similar to $M=\left[\begin{array}{rr}2 & 0 \\ 0 & -2\end{array}\right]$ and $\left[\begin{array}{ll}0 & 2 \\ 2 & 0\end{array}\right]$. For $x^{2}+4 x+4$, we have $M=\left[\begin{array}{rr}-2 & 0 \\ 0 & -2\end{array}\right]$ and for $x^{2}-4 x+4$, we have $M=\left[\begin{array}{ll}2 & 0 \\ 0 & 2\end{array}\right]$. 
$|\operatorname{det}(A)|=5$. In this case, $m^{2}+n^{2}=5$ if and only if $m= \pm 1, n= \pm 2$ or $m= \pm 2, n=$ \pm 1 . Therefore, we need to consider the expanding polynomials: $x^{2} \pm 5, x^{2} \pm 2 x+5$, $x^{2} \pm 4 x+5$. For $x^{2}+5, x^{2} \pm 2 x+5, C \nsucc M$ by Corollary 7.3 since $v \neq 2 k$ with nonzero $k$. In fact, for the characteristic polynomial $x^{2}+5, A \sim M=\left[\begin{array}{cc}m & n \\ -n & m\end{array}\right]$ implies that $m=0$ since the trace is zero, which is impossible. Thus, no $A$ with characteristic polynomial $x^{2}+5$ is $\mathbb{Z}$-similar to a self-similar matrix. For $x^{2}+2 x+5, C \nsucc M=\left[\begin{array}{rr}-1 & 2 \\ -2 & -1\end{array}\right]$ and for $x^{2}-2 x+5, C \nsucc M=\left[\begin{array}{rr}1 & 2 \\ -2 & 1\end{array}\right]$ by Table 4. For $x^{2}-5, C \sim\left[\begin{array}{lr}2 & 1 \\ 1 & -2\end{array}\right]$ by the remark. The other self-similar matrix for $x^{2}-5$ is $\left[\begin{array}{rr}1 & 2 \\ 2 & -1\end{array}\right] \nsim C$ by Table 4 . For $x^{2}+4 x+5$, there is only one $\mathbb{Z}$-similar class and we have $C \sim\left[\begin{array}{ll}-2 & 1 \\ -1 & -2\end{array}\right]$. Similarly, for $x^{2}-4 x+5$, we have $C \sim\left[\begin{array}{rr}2 & 1 \\ -1 & 2\end{array}\right]$.

\section{Acknowledgment}

The authors thank Professor Y. Wang for introducing this topic while he was visiting the Institute of Mathematical Sciences (IMS) in CUHK. They also thank Dr. Hui Rao for some suggestions concerning the standard digit sets.

\section{Appendix}

In Table 2 we list the characteristic polynomials for the $2 \times 2$ expanding integer matrices $A$ with $|\operatorname{det}(A)|=2,3,4,5$ (Proposition 2.6). Table 3 involves such c.p. that have more than one $\mathbb{Z}$-similar class besides the companion matrices.

Finally, using the results in Section 7, we list those characteristic polynomials in Table 4 that have self-similar representatives.

Table 2. Characteristic polynomials.

\begin{tabular}{llll}
\hline$|\operatorname{det}(A)|=2$ & $|\operatorname{det}(A)|=3$ & $|\operatorname{det}(A)|=4$ & $|\operatorname{det}(A)|=5$ \\
\hline$x^{2} \pm 2$ & $x^{2} \pm 3$ & $x^{2} \pm 4$ & $x^{2} \pm 5$ \\
$x^{2} \pm x+2$ & $x^{2} \pm x-3$ & $x^{2} \pm x-4$ & $x^{2} \pm x-5$ \\
$x^{2} \pm 2 x+2$ & $x^{2} \pm x+3$ & $x^{2} \pm 2 x-4$ & $x^{2} \pm 2 x-5$ \\
& $x^{2} \pm 2 x+3$ & $x^{2} \pm x+4$ & $x^{2} \pm 3 x-5$ \\
& $x^{2} \pm 3 x+3$ & $x^{2} \pm 2 x+4$ & $x^{2} \pm x+5$ \\
& & $x^{2} \pm 3 x+4$ & $x^{2} \pm 2 x+5$ \\
& & $x^{2} \pm 4 x+4$ & $x^{2} \pm 3 x+5$ \\
& & & $x^{2} \pm 4 x+5$ \\
& & & $x^{2} \pm 5 x+5$
\end{tabular}


Table 3. Representatives of the nonunique $\mathbb{Z}$-similar classes.

\begin{tabular}{|c|c|c|c|}
\hline $\begin{array}{l}\text { Characteristic } \\
\text { polynomials of } A\end{array}$ & $\begin{array}{c}\text { Number of } \\
\text { similarity classes }\end{array}$ & \multicolumn{2}{|c|}{$\begin{array}{l}\text { Representatives of } \\
\text { classes besides C }\end{array}$} \\
\hline$x^{2}+3$ & 2 & {$\left[\begin{array}{r}1 \\
-2\end{array}\right.$} & $\left.\begin{array}{r}2 \\
-1\end{array}\right]$ \\
\hline$x^{2}-2 x-4$ & 2 & {$\left[\begin{array}{l}0 \\
2\end{array}\right.$} & $\left.\begin{array}{l}2 \\
2\end{array}\right]$ \\
\hline$x^{2}-4$ & 3 & {$\left[\begin{array}{rr}2 & n \\
0 & -2\end{array}\right]$,} & $n=0,2$ \\
\hline$x^{2}+2 x-4$ & 2 & {$\left[\begin{array}{l}0 \\
2\end{array}\right.$} & $\left.\begin{array}{r}2 \\
-2\end{array}\right]$ \\
\hline$x^{2}-4 x+4$ & $\infty$ & {$\left[\begin{array}{rr}2 & |n| \\
0 & 2\end{array}\right]$} & $|n| \neq 1$ \\
\hline$x^{2}-2 x+4$ & 2 & {$\left[\begin{array}{r}0 \\
-2\end{array}\right.$} & $\left.\begin{array}{l}2 \\
2\end{array}\right]$ \\
\hline$x^{2}-x+4$ & 2 & {$\left[\begin{array}{l}-1 \\
-3\end{array}\right.$} & $\left.\begin{array}{l}2 \\
2\end{array}\right]$ \\
\hline$x^{2}+4$ & 2 & {$\left[\begin{array}{r}0 \\
-2\end{array}\right.$} & $\left.\begin{array}{l}2 \\
0\end{array}\right]$ \\
\hline$x^{2}+x+4$ & 2 & {$\left[\begin{array}{r}1 \\
-3\end{array}\right.$} & $\left.\begin{array}{r}2 \\
-2\end{array}\right]$ \\
\hline$x^{2}+2 x+4$ & 2 & {$\left[\begin{array}{r}0 \\
-2\end{array}\right.$} & $\left.\begin{array}{r}2 \\
-2\end{array}\right]$ \\
\hline$x^{2}+4 x+4$ & $\infty$ & {$\left[\begin{array}{rr}-2 & |n| \\
0 & -2\end{array}\right]$} &,$\quad|n| \neq 1$ \\
\hline$x^{2}-2 x-4$ & 2 & {$\left[\begin{array}{l}0 \\
2\end{array}\right.$} & $\left.\begin{array}{l}2 \\
2\end{array}\right]$ \\
\hline$x^{2}-5$ & 2 & {$\left[\begin{array}{l}1 \\
2\end{array}\right.$} & $\left.\begin{array}{r}2 \\
-1\end{array}\right]$ \\
\hline$x^{2}+2 x+5$ & 2 & {$\left[\begin{array}{l}-1 \\
-2\end{array}\right.$} & $\left.\begin{array}{r}2 \\
-1\end{array}\right]$ \\
\hline$x^{2}-2 x+5$ & 2 & {$\left[\begin{array}{r}1 \\
-2\end{array}\right.$} & $\left.\begin{array}{l}2 \\
1\end{array}\right]$ \\
\hline$x^{2}+5$ & 2 & {$\left[\begin{array}{r}2 \\
-3\end{array}\right.$} & $\left.\begin{array}{r}3 \\
-2\end{array}\right]$ \\
\hline
\end{tabular}


Table 4. $\mathbb{Z}$-similar classes with self-similar representatives.

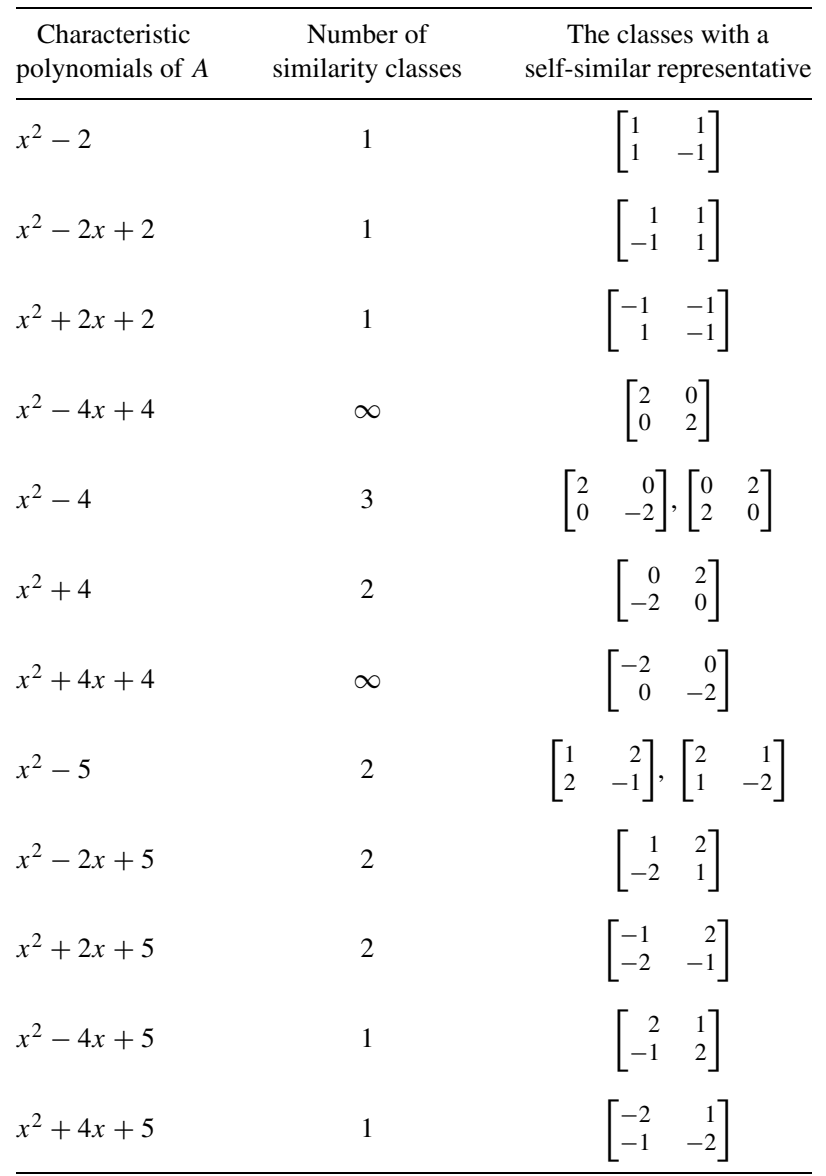

\section{References}

[B] C. Bandt, Self-similar sets, 5. Integer matrices and fractal tilings of $\mathbb{R}^{n}$, Proc. Amer. Math. Soc. 112 (1991), 549-562.

[BG] C. Bandt and G. Gelbrich, Classification of self-affine lattice tilings, J. London Math. Soc. 50 (1994), 581-593.

[F] K. Falconer, Fractal Geometry, Mathematical Foundations and Applications, Wiley, New York, 1990.

[GH] K. Gröchenig and A. Haas, Self-similar lattice tilings, J. Fourier Anal. Appl. 1 (1994), 131-170.

[GM] K. Gröchenig and W. Madych, Multiresolution analysis, Haar bases, and self-similar tilings, IEEE Trans. Inform. Theory 38 (1992), 556-568.

[H] J. Hutchinson, Fractals and self-similarity, Indiana Univ. Math. J. 30 (1981), 713-747.

[HSV] D. Hacon, N. C. Saldanha, and J. J. P. Veerman, Remarks on self-affine tilings, Experimental Math. 3 (1994), 317-327.

[KL] I. Kirat and K. S. Lau, On the connectedness of integral self-affine tiles, J. London Math. Soc. (2) 62 (2000), 291-304.

[KLSW] R. Kenyon, J. Li, R. S. Strichartz, and Y. Wang, Geometry of self-affine tiles, II, Indiana Univ. Math. J. 48 (1999), 24-42. 
[LW1] J. C. Lagarias and Y. Wang, Haar-type orthonormal wavelet bases in $\mathbb{R}^{2}$, J. Fourier Anal. Appl. 2 (1995), 1-14

[LW2] J. C. Lagarias and Y. Wang, Self-affine tiles in $\mathbb{R}^{n}$, Adv. in Math. 121 (1996), 21-49.

[LW3] J. C. Lagarias and Y. Wang, Integral self-affine tiles in $\mathbb{R}^{n}$, I. Standard and non-standard digit sets, J. London Math. Soc. 54 (1996), 161-179.

[LW4] J. C. Lagarias and Y. Wang, Haar bases for $L^{2}\left(\mathbb{R}^{2}\right)$ and algebraic number theory, J. Number Theory 57 (1996), 181-197.

[LW5] J. C. Lagarias and Y. Wang, Integral self-affine tiles in $\mathbb{R}^{n}$, II. Lattice tiling, J. Fourier Anal. Appl. 3 (1997), 1-20.

[Ma] D. Marcus, Number Fields, Springer-Verlag, New York, 1977.

[Mo] R. A. Mollin, Quadratics, CRC Press, Boca Raton, FL, 1996.

[N] M. Newman, Integer Matrices, Academic Press, New York, 1972.

[SW] R. S. Strichartz and Y. Wang, Geometry of self-affine tiles, I, Indiana Univ. Math. J., 48 (1999), 1-23.

Received October 30, 2000. Online publication April 19, 2002. 